\title{
Wedge indentation of single crystalline monazite: A numerical investigation
}

\author{
Juul, K. J.; Nellemann, C.; Nielsen, K.L.; Niordson, C. F.; Kysar, J. W.
}

Published in:

International Journal of Plasticity

Link to article, DOI:

10.1016/j.ijplas.2018.08.005

Publication date:

2019

Document Version

Peer reviewed version

Link back to DTU Orbit

Citation (APA):

Juul, K. J., Nellemann, C., Nielsen, K. L., Niordson, C. F., \& Kysar, J. W. (2019). Wedge indentation of single crystalline monazite: A numerical investigation. International Journal of Plasticity, 112, 36-51.

https://doi.org/10.1016/j.ijplas.2018.08.005

\section{General rights}

Copyright and moral rights for the publications made accessible in the public portal are retained by the authors and/or other copyright owners and it is a condition of accessing publications that users recognise and abide by the legal requirements associated with these rights.

- Users may download and print one copy of any publication from the public portal for the purpose of private study or research.

- You may not further distribute the material or use it for any profit-making activity or commercial gain

- You may freely distribute the URL identifying the publication in the public portal 


\section{Accepted Manuscript}

Wedge indentation of single crystalline monazite: A numerical investigation

K.J. Juul, C. Nellemann, K.L. Nielsen, C.F. Niordson, J.W. Kysar

PII: $\quad$ S0749-6419(18)30390-5

DOI: $\quad$ 10.1016/j.ijplas.2018.08.005

Reference: INTPLA 2395

To appear in: International Journal of Plasticity

Received Date: 8 July 2018

Accepted Date: 10 August 2018

Please cite this article as: Juul, K.J., Nellemann, C., Nielsen, K.L., Niordson, C.F., Kysar, J.W., Wedge indentation of single crystalline monazite: A numerical investigation, International Journal of Plasticity (2018), doi: 10.1016/j.jplas.2018.08.005.

This is a PDF file of an unedited manuscript that has been accepted for publication. As a service to our customers we are providing this early version of the manuscript. The manuscript will undergo copyediting, typesetting, and review of the resulting proof before it is published in its final form. Please note that during the production process errors may be discovered which could affect the content, and all legal disclaimers that apply to the journal pertain. 


\title{
Wedge indentation of single crystalline monazite: A numerical investigation
}

\author{
K. J. Juul ${ }^{\mathrm{a}, *}$, C. Nellemann ${ }^{\mathrm{a}}$, K. L. Nielsen ${ }^{\mathrm{a}}$, C. F. Niordson ${ }^{\mathrm{a}}$, J. W. Kysar ${ }^{\mathrm{b}}$ \\ ${ }^{a}$ Department of Mechanical Engineering, Solid Mechanics, Technical University of \\ Denmark, DK-2800 Kgs. Lyngby, Denmark \\ ${ }^{b}$ Department of Mechanical Engineering, Columbia University, New York, NY 10027, \\ United States
}

\section{Abstract}

A numerical investigation of wedge indentation, with a nearly flat indenter, into a monazite $\left(\mathrm{LaPO}_{4}\right)$ single crystal is carried out to obtain the asymptotic field solution associated with the moving contact point singularities. The crystal orientation is such that plane strain conditions prevail, under the assumption of small scale yielding, as out-of-plane deformations are eliminated due to out-of-plane mirror symmetry of the crystal, specimen and loading state. The plastic deformation in such a 2D study can be described in terms of effective in-plane slip systems comprised of crystallographic slip systems with equal and opposite out-of-plane deformation and rotation. The numerical simulations are conducted within a framework specialized for selfsimilar problems and adopts a visco-plastic single crystal material model. The detailed numerical investigation of the monazite single crystal reveals that the effective slip systems lead to a non-symmetric in-plane deformation

\footnotetext{
${ }^{*}$ Corresponding author

Email address: krjoju@mek.dtu.dk (K. J. Juul)
} 
field, which is consistent with the absence of in-plane mirror symmetries of the crystal. Interestingly, the non-symmetric deformation field results in one contact point singularity travelling at a greater speed than the other. The deformation near the moving contact point singularities are found to be divided into two angular sectors separated by a boundary of glide shear type. The slip rates on the individual systems reveal that one slip system dominates at both contact points, whereas the other slip system shows negligible activity. Thus, only one slip system gives rise to a discontinuity in the slip rate field.

Keywords: Self-similarity, Crystal plasticity, Monoclinic structure, Asymptotic fields

\section{Introduction}

Naturally occurring monazite may be recognized as a reddish-brown phosphate mineral. Monazite is usually found in small isolated crystals and con4 tains rare earth metals such as cerium, lanthanum, and neodymium. A par5 ticular interest in the lanthanum phosphate $\left(\mathrm{LaPO}_{4}\right)$ monazite material exists 6 due to its relatively low hardness, high-temperature stability, and compatibil7 ity with common structural oxide ceramics, while exhibiting weak bonding to other oxide elements. These special mechanical properties make monazite an

ideal material for fiber reinforced ceramic matrix composites. For example, monazite coated fibers have proven to hinder destructive damage mechanisms by enabling crack deflection. Furthermore, the high-temperature sta- 
bility eliminates the problem of oxidation which is commonly observed during fiber pullout for interface materials used in ceramic matrix composites (Davis et al., 2003; Ruggles-Wrenn et al., 2009).

Investigation of the asymptotic slip solution field near moving singular points (such as growing cracks and wedge indentation), based on slip-line theory that assumes linear elastic, perfectly plastic material behaviour, dates back to the 1980s (Drugan et al., 1982; Drugan and Rice, 1984; Drugan, 1986; Rice, 1987; Mesarovic and Kysar, 1996; Drugan, 2001; Kysar, 2001a,b; Saito and Kysar, 2011). The early studies predict that the deforming domain near a moving contact point singularity can be divided into sectors separated by glide or kink shear discontinuities. The deformation, within each sector, will be governed by either purely elastic or active plastic deformation on a number of slip systems. The fundamental properties of asymptotic fields, in relation to both fracture and wedge indentation in single crystals, have been investigated experimentally by a number of authors (Bastawros and Kim, 1998; Kysar, 2000; Crone and Shield, 2001; Kysar and Briant, 2002; Kysar et al., 2010; Saito and Kysar, 2011; Saito et al., 2012; Dahlberg et al., 2014; Sarac et al., 2016; Dahlberg et al., 2017; Juul et al., 2018; Sarac and Kysar, 2018). However, existing studies are limited to the three most common crystal structures being FCC, BCC, and HCP. In fact, only few studies investigate the details of the deformation in more special crystal configurations such as the monazite crystal (monoclinic structure). The existing numerical investigations of monazite have focused mainly on determining anisotropic 
elastic parameters, thermal conductivity (Feng et al., 2013), and radiation resistance (Grechanovsky et al., 2013). Furthermore, the deformation mechanisms (experimentally observed active slip systems and twinning) of polycrystalline monazite have been investigated by Hay and Marshall (2003) and Hay $(2005,2008)$.

The aim of the present study is to investigate the deformation field, and the associated asymptotic fields in the vicinity of moving contact singularities for wedge indentation (i.e. the point where the indenter continuously comes into contact with new surface material), with a nearly flat indenter, into an elastic, perfectly plastic monazite single crystal. The results will serve as foundation for understanding the properties of monazite measured in indentation as the asymptotic solution reveals how dislocation activity can be linked to the formation of kink shear sector boundaries.

The paper is divided into the following sections: The wedge indentation problem is outlined in Section 2. The material model, derivation of the inplane effective slip systems and the yield surface of monazite are presented in Section 3. The numerical framework is presented in Section 4, and results are presented in Section 5. Concluding remarks are given in Section 6 . Throughout, index notation, including Einstein's summation convention, is used and a superimposed dot, $\left({ }^{\circ}\right)$, signifies the time derivative. 


\section{Wedge indentation with a nearly flat indenter}

Wedge indentation into a monazite single crystal is simulated along the lines of Saito et al. (2012) and Juul et al. (2018) with a nearly flat indenter such that the indenter angle, $\phi$, is close to zero degrees (see Fig. 1). Thus, a small strain assumption is valid. Furthermore, the indentation is performed under the assumption of negligible friction between the rigid indenter and an elastic, perfectly plastic material with a very low yield resistance (model parameters are listed in Table 1). The study is performed under such conditions to investigate the asymptotic field in the vicinity of the moving contact points as well as to ensure a direct comparison to work existing on more common crystal structures (Saito and Kysar, 2011; Saito et al., 2012; Juul et al., 2018).

In a corresponding numerical study, Juul et al. (2018) compared results for the FCC and BCC crystal structures to the analytical predictions of Saito and Kysar (2011), which are based on an extension of slip line theory that assumes a linear elastic, perfectly plastic material behavior. Good agreement was found for this comparison. Following Rice (1987), the analytical investigation by Saito and Kysar (2011) showed that the asymptotic deformation fields consist of angular sectors centered at the moving contact point singularity, with the sectors deforming either elastically or plastically. The angular sectors are separated by radial rays, emanating from the moving contact point, that coincide either with the slip direction or the slip plane normal of the slip systems. As described by Rice (1987), if the radial ray coincides with 
a slip direction, dislocations operate in glide shear along the ray, and if the radial ray coincides with the slip plane normal, dislocations operate in kinkshear mode. As the contact point singularity moves quasistatically relative to the crystal, the angular sectors and sector boundaries move correspondingly through the crystal, hence, velocity discontinuities across the radial sector boundaries exist under these conditions (Drugan and Rice, 1984). Both glide shear and kink shear sector boundaries have been identified experimentally (Bastawros and Kim, 1998; Crone and Shield, 2001; Kysar and Briant, 2002; Kysar et al., 2010).

The driving force behind the present study is that such analytical solutions do not currently exist for the monoclinic crystal structure of monazite, but it is expected that the asymptotic solution near the moving points is governed by the same characteristics as the analytical solutions for FCC and BCC crystals. To bring out the angular sectors and sector boundaries for monazite crystals, the stress and slip rate distributions near the moving contact point singularity are analyzed in detail (see Section 5). Due to the velocity discontinuities, that are expected in the present study, spikes of highly localized slip rate emanating from the contact points are also investigated.

\section{Monazite single crystals}

\subsection{Material model}

The wedge indentation study is conducted in a plane strain setting with a small strain formulation, where the displacement field, $u_{i}$, provides the 
total strain, $\varepsilon_{i j}$, through the relation; $\varepsilon_{i j}=\left(u_{i, j}+u_{j, i}\right) / 2$. The total strain is decomposed into an elastic part, $\varepsilon_{i j}^{e}$, and a plastic part, $\varepsilon_{i j}^{p}$, such that $\varepsilon_{i j}=\varepsilon_{i j}^{e}+\varepsilon_{i j}^{p}$. When the elastic strain field is known, the stress field can be determined by; $\sigma_{i j}=\mathscr{L}_{i j k l} \varepsilon_{k l}^{e}$, where $\mathscr{L}_{i j k l}$ is the elastic stiffness tensor.

The elastic strains, required for the calculation of stresses, are determined by obtaining the plastic strains through summation over all slip systems, $\alpha$ :

$$
\dot{\varepsilon}_{i j}^{p}=\sum_{\alpha} \dot{\gamma}^{(\alpha)} P_{i j}^{(\alpha)}, \quad P_{i j}^{(\alpha)}=\frac{1}{2}\left(s_{i}^{(\alpha)} m_{j}^{(\alpha)}+m_{i}^{(\alpha)} s_{j}^{(\alpha)}\right) .
$$

Here, $P_{i j}^{(\alpha)}$ is the Schmid tensor, $\dot{\gamma}^{(\alpha)}$ is the slip rate, and $s_{i}^{(\alpha)}$ and $m_{i}^{(\alpha)}$ are the unit vectors defining the slip direction and the slip plane normal, respectively (slip direction and normal are illustrated in Fig. 2b). The slip rate on the individual slip systems, denoted by superscript, $\alpha$, is determined by employing the visco-plastic power law slip rate relation proposed by Hutchinson (1976)

$$
\dot{\gamma}^{(\alpha)}=\dot{\gamma}_{0} \operatorname{sgn}\left(\tau^{(\alpha)}\right)\left(\frac{\left|\tau^{(\alpha)}\right|}{\tau_{0}^{(\alpha)}}\right)^{1 / m}
$$

where $\tau^{(\alpha)}=\sigma_{i j} m_{i}^{(\alpha)} s_{j}^{(\alpha)}$ is the resolved shear stress, $\tau_{0}^{(\alpha)}$ is the critically resolved shear stress, and $m$ is the rate sensitivity exponent (not to be confused with the slip normal $\left.m_{i}^{(\alpha)}\right)$. The present study concerns the rate independent limit and thus the rate sensitivity exponent is pushed towards this limit $(m \rightarrow 0)$ 


\subsection{In-plane slip systems for the 2D study}

The monazite material is monoclinic in crystal structure and belongs to the $P 2_{1} / n$ space group. The primitive monoclinic lattice structure is characterized accordingly in the so-called second setting (Donnay, 1943; Matthies and Wenk, 2009), with lattice parameters; $a=0.6825 \mathrm{~nm}, b=0.7057 \mathrm{~nm}$, $c=0.6482 \mathrm{~nm}$, and $\beta=103.21^{\circ}\left(\angle b c=\angle a b=90^{\circ}\right)$ as sketched in Fig. 2a (Hirth and Lothe, 1968; Hay, 2008). The low symmetry and large lattice parameters, compared to common metals with FCC and BCC structures, make the characterization of the material deformation somewhat more complex. For the monoclinic crystal structure the slip plane normal, given by the Miller-index representation ( $\mathrm{hkl}$ ), is associated with the non-orthogonal basis of the reciprocal lattice vectors and the slip direction is given by the Miller-index representation [hkl] associated with the non-orthogonal basis of the lattice vectors.

Plane strain conditions in a single crystal can be achieved by choosing the plane of deformation such that it coincides with a mirror symmetry plane in the crystal (see e.g. Rice, 1987; Crone et al., 2004; Kysar et al., 2005; Niordson and Kysar, 2014). Moreover, it is a requirement that the specimen geometry and the external loading also possess mirror symmetry with respect to the crystal mirror symmetry plane. Complying with these conditions, the slip systems can be arranged into mirrored pairs, such that each slip system of the pair has an identical resolved shear stress. The mirrored pair of slip systems are then assumed to active in equal amounts when at the 
critical resolved shear stress. Any out-of-plane plastic deformation of one slip system is counteracted by the other slip system of the pair. Hence, the net deformation state of the mirrored pair can be described by a single effective in-plane slip system.

Hay (2008) presents findings of active slip systems in monazite and 12 slip systems of interest are identified as possible candidates to form mirrored pairs of slip systems. These slip systems are summarized in Table 2. The identified slip systems, however, do not reflect the possible difference in slip resistance along opposite directions of slip. However, based on the discussion of this issue by Hay (2008), the difference is assumed negligible in the present work.

Figure 2a presents the definition of the crystallographic orientation in three dimensional space (a Cartesian orthonormal basis). Here, the crystallographic orientation of the monazite is chosen such that the (010) plane is the mirror symmetry plane for the plane strain deformation (see Fig. 2c). When interpreting Figure 2, one should be aware of the following:

1. The lattice vector $b$ is parallel to the [010] direction, the $x_{2}$-axis and the (010) plane normal.

2. The lattice vector $c$ is parallel to the [001] direction and the $x_{3}$-axis.

3. The lattice vector $a$ is parallel to the [100] direction and the $(010) \times$ (001) direction, thus, the lattice vector is oriented in the $x_{1} x_{3}$-plane (mirror plane) at the angle $\beta$ with respect to the $x_{3}$-axis (clockwise rotation). 
4. The reciprocal lattice vector represented by the (100) plane normal is parallel to the $x_{1}$-axis.

5. The reciprocal lattice vector represented by the (001) plane normal is parallel to the direction $[100] \times[010]$.

For monazite single crystals, in the chosen configuration, three in-plane slip systems can be identified. Two being effective slip systems (mirrored pairs of crystallographic slip systems), and one being a crystallographic slip system in itself. The first in-plane slip system (Fig. 2d) is comprised of the two crystallographic slip systems (011)[100] and (011)[100] that are equivalent to the effective slip system (001)[100]. The second in-plane system (Fig. 2e) is the crystallographic slip system (100)[001] which coincides with the third in-plane slip system (Fig. 2f), constructed from the crystallographic

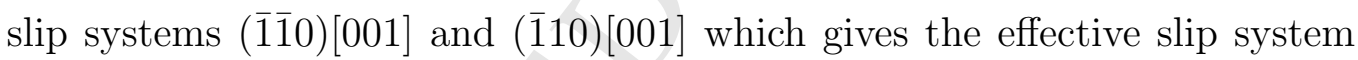
(100)[001]. It is seen that the first in-plane slip direction is at an angle of $-103.21^{\circ}$ with respect to the $x_{3}$-axis, while the second and the third inplane slip directions (one crystallographic and one effective) are parallel to the $x_{3}$-axis. The three in-plane slip systems are summarized in Table 3 by in-plane unit vectors $S_{I}^{(A)}$ and $M_{I}^{(A)}$, which are the rescaled projections of $s_{i}^{(\alpha)}$ and $m_{i}^{(\alpha)}$ onto the $x_{1} x_{3}$ mirror symmetry plane, respectively. Here, the superscript represents the in-plane slip systems and the subscript represents in-plane index notation. Lower case letters represent the actual slip systems and upper case letters represent the in-plane systems. Furthermore, the in-plane unit vectors $s_{i}^{(\alpha)}$ and $m_{i}^{(\alpha)}$ will be substituted for $S_{I}^{(A)}$ and $M_{I}^{(A)}$, 


\subsection{Yield surface}

Yielding is assumed to be governed by the Schmid law through the resolved shear stress

$$
\sigma_{i j} m_{i}^{(\alpha)} s_{j}^{(\alpha)}= \pm \tau_{0} \quad \text { for } \quad i, j \in\{1,2,3\}
$$

with the superscript $(\alpha)$ omitted on the critically resolved shear stress since this value is assumed equal for all crystallographic slip systems.

Considering plane strain loading in the mirror symmetry plane of the monazite crystal as (010), the stress tensor has no out-of-plane shear $\left(\sigma_{12}=\right.$ $\sigma_{32}=0$ ) and Eq. (3) reduces to

$$
\left(s_{1}^{(\alpha)} m_{3}^{(\alpha)}+s_{3}^{(\alpha)} m_{1}^{(\alpha)}\right) \sigma_{13}+2 s_{1}^{(\alpha)} m_{1}^{(\alpha)}\left(\frac{\sigma_{11}-\sigma_{33}}{2}\right)= \pm \tau_{0} .
$$

showing that there is no dependence on the out-of-plane normal stress $\sigma_{22}$.

Based on the geometric constraints governing the in-plane slip direction vector, slip plane normal vector, and the definition of the in-plane angle $\theta^{(A)}$ (with a positive rotation defined counter clockwise, see Fig. 2b), the relations $S_{1}^{(A)}=\cos \left(\theta^{(A)}\right)=M_{3}^{(A)}$ and $S_{3}^{(A)}=\sin \left(\theta^{(A)}\right)=-M_{1}^{(A)}$ permit Eq. (4) to be expressed as

$$
\sigma_{13}=\tan \left(2 \theta^{(A)}\right)\left(\frac{\sigma_{11}-\sigma_{33}}{2}\right) \pm \frac{\lambda^{(A)} \tau_{0}}{\cos \left(2 \theta^{(A)}\right)}
$$


Here, $\lambda^{(A)}=\frac{\tau_{0}^{(A)}}{\tau_{0}}$ is the ratio of slip resistance on the effective slip system (A) and the crystallographic slip system. For the FCC and the BCC crystal structures, Rice (1987); Niordson and Kysar (2014); Juul et al. (2017, 2018) have discussed the scaling ratios associated with slip system properties that are needed for the response of the effective slip systems and the crystallographic slip systems to coincide under plane strain conditions. In the present study, scaling of the critically resolved shear stress and scaling of the reference strain rate will be sufficient and result in the following relations

$$
\tau_{0}^{(A)}=\lambda^{(A)} \tau_{0}, \quad \text { and } \quad \dot{\gamma}_{0}^{(A)}=\beta^{(A)} \dot{\gamma}_{0}
$$

The scaling ratios for each of the effective slip systems are listed in Table 3, which also include expressions for the two quantities based on slip system unit vectors (both effective and crystallographic unit vectors). As seen from Table 3, the scaling ratios of the two effective in-plane slip systems (1) and (3) are larger than unity (being the scaling factor for effective slip system (2)). The expression for the yield surface in Eq. (5), normalized with the critically resolved shear stress, allows for a convenient representation of the yield criterion in a two dimensional stress space with abscissa $\left(\sigma_{11}-\sigma_{33}\right) /\left(2 \tau_{0}\right)$ and ordinate $\sigma_{13} / \tau_{0}$ (also discussed by Kysar et al., 2005). The yield surface thereby reveals itself as three sets of parallel lines, oriented at the angle $2 \theta^{(A)}$ with respect to the $\left(\sigma_{11}-\sigma_{33}\right) /\left(2 \tau_{0}\right)$-axis, the distance $\lambda^{(A)}$ (perpendicular to the parallel lines) from the origin in normalized stress space. The inner 
envelope described by the parallel lines of the slip systems (1) and (2), and the outer envelope described by parallel lines of slip systems (1) and (3) form yield surfaces with the vertices listed in Table 4. An illustration of the yield surface is shown in Fig. 3 where it forms a parallelogram with the vertices A, $\mathrm{B}, \mathrm{C}$ and $\mathrm{D}$ in normalized stress space. In case of the two sets of parallel slip systems (one effective and one crystallographic), the scaling factor $\lambda^{(2)}$ results in the lowest critically resolved shear stress, such that plastic deformation will take place on in-plane slip system (2) for the elastic, perfectly plastic material considered. Thus, the effective in-plane slip system (3) (having the highest slip resistance) is assumed inactive in the present study and only slip system (1) and (2) are included in the numerical analysis (obviously this argument would not suffice in the case of a hardening solid).

\section{Numerical framework}

\subsection{Self-similarity}

The present work employs a numerical framework developed for selfsimilar problems, where the features of the field quantities remain unchanged, while the fields change in size over time. This framework was first presented in Juul et al. (2018) and employed to wedge indentation in FCC, BCC, and HCP single crystals with excellent results. It has the great advantages over conventional temporal finite element approaches in that: 1) the contact point remains stationary relative to the finite element mesh (avoiding discrete nodal contact events) and thus inherently captures the continuously increasing con- 
tact area6; and 2) a highly refined mesh can easily be focused at the contact points as they do not move in this computational framework.

For wedge indentation, the self-similar condition is achieved when the indentation rate, $\dot{a} / a=\dot{c}$ (with $a$ being the half contact length and $\dot{a}$ being the contact velocity), is constant. However, compared to the study of Juul et al. (2018), indentation in a monazite crystal is slightly different due to the non-symmetric nature of the monoclinic structure. The FCC, BCC, and HCP crystals, all studied in Juul et al. (2018), gave rise to a symmetric displacement field, such that the distance from the indenter tip to the moving contact point singularity on both sides of the indenter tip are equal. However, this is not the case for monazite. Due to the monoclinic crystal structure, the displacement field will not remain symmetric and, thus, the velocity of the contact points to the left and right of the indenter tip will be different. Therefore, it is necessary to introduce individual contact lengths (and velocities) for the left and right contact points, respectively. These will be denoted $a_{r}$ and $a_{l}$ as illustrated in Fig. 1. To satisfy the condition $\dot{a} / a=\dot{c}$, that ensures a self-similar solution, the quantities $a$ and $\dot{a}$ will then have to be treated as the average values such that; $a=\left(a_{r}+a_{l}\right) / 2$ and $\dot{a}=\left(\dot{a}_{r}+\dot{a}_{l}\right) / 2$.

The self-similar development of the solution in indentation can be recognized by an observer changing the magnification of the view at a rate related to the indentation process, such that the field quantities appear constant in both shape and size. This noteworthy characteristic of self-similar problems ties to the fact that only one independent characteristic length exists. Thus, 
the only time dependence in the problem enters through the evolution of this characteristic length, $a$ (see discussion in Juul et al., 2018). The time rate of change of any field quantity can thereby be related to a corresponding spatial derivative in the self-similar coordinate system through the relation

$$
\dot{f}=-\dot{c} \xi_{i} \frac{\partial f}{\partial \xi_{i}}
$$

where $\dot{c}$ is the indentation rate and $\xi_{i}$ is a self-similar coordinate system, where the coordinates of the material points change with time. Based on this key relation between time and spatial derivatives, it is possible to obtain the history dependence through spatial integration when solving the problem. The numerical scheme for the spatial integration is adopted from Juul et al. (2018). Here, the integration lines are also located radially around the indenter tip (see Fig. 1), making it convenient to express the self-similar relation (Eq. (7)) in a self-similar polar coordinate system with the origin located at the tip of the indenter. The self-similar relation thereby transforms into

$$
\dot{f}=-\dot{c} \rho \frac{\partial f}{\partial \rho}
$$

where $\rho$ is the radial distance to a point on the integration line.

\subsection{Numerical implementation}

The self-similar relation in Eq. (8) is applied to the material model outlined in Section 3.1. Thus, all time derivatives are transformed into corre- 
sponding spatial derivatives through the indentation rate, $\dot{c}$, and the distance to the indenter tip, $\rho$. A quantity of interest at the material point, $\rho^{*}$, can be envisioned to lie on a line that ends on the indenter tip and its deformation history is accounted for by spatial integration along that line toward the tip. Essentially, the integration starts in the elastic region (say point $\rho^{0}$ ) far from the indenter tip, and ends at the point of interest, $\rho^{*}$ (see Fig. 1). Thus, the point of interest, $\rho^{*}$, obtains the history through information stored in points further away from the indenter tip. This integration method ensures that the elastic-plastic material response of the material is captured and also allows for potential elastic unloading.

Other than this tailored history integration, the framework adopted from Juul et al. (2018), relies on the conventional principle of virtual work (PVW) for a quasi-static problem to determine the displacement field, $u_{i}$;

$$
\int_{V} \mathscr{L}_{i j k l} \varepsilon_{k l} \delta \varepsilon_{i j} \mathrm{~d} V=\int_{S} T_{i} \delta u_{i} \mathrm{~d} S+\int_{V} \mathscr{L}_{i j k l} \varepsilon_{k l}^{p} \delta \varepsilon_{i j} \mathrm{~d} V
$$

Here, $T_{i}=\sigma_{i j} n_{j}$ is the surface traction $\left(n_{j}\right.$ denotes the unit outward normal vector), $V$ is the volume of the domain, and $S$ is the boundary of the domain. The PVW is solved by employing the finite element method with 2D 8-node isoparametric elements using reduced Gauss integration $(2 \times 2$ Gauss points $)$.

The implementation of the self-similar framework follows Juul et al. (2018), and the pseudo-algorithm is as follows:

1. Determine the displacement field, $u_{i}^{(n)}$, by use of the plastic strains 
from the previous iteration, $\varepsilon_{i j}^{p(n-1)}$ ( $n$ is the iterative step).

2. Determine the total strains, $\varepsilon_{i j}^{(n)}$ from the current displacement field, $u_{i}^{(n)}$.

3. Initiate spatial integration scheme to determine the slip and plastic strains.

(a) The spatial derivatives are determined by applying the self-similar relation in Eq. (8) to the rate equations in the constitutive law.

$$
\begin{aligned}
\frac{\partial \gamma^{(\alpha)}}{\partial \rho} & =-\frac{\dot{\gamma}_{0}^{(\alpha)}}{\rho \dot{c}} \operatorname{sgn}\left(\tau^{(\alpha)}\right)\left(\frac{\left|\tau^{(\alpha)}\right|}{g^{(\alpha)}}\right)^{1 / m} \\
\frac{\partial \varepsilon_{i j}^{p}}{\partial \rho} & =\sum_{\alpha} \frac{\partial \gamma^{(\alpha)}}{\partial \rho} P_{i j}^{(\alpha)}
\end{aligned}
$$

(b) The current slip, $\gamma^{(\alpha)(n)}$, and plastic strains, $\varepsilon_{i j}^{p(n)}$, are determined through spatial integration

$$
\gamma^{(\alpha)(n)}=\int_{\rho^{0}}^{\rho^{*}} \frac{\partial \gamma^{(\alpha)}}{\partial \rho} \mathrm{d} \rho, \quad \text { and } \quad \varepsilon_{i j}^{p(n)}=\int_{\rho^{0}}^{\rho^{*}} \frac{\partial \varepsilon_{i j}^{(p)}}{\partial \rho} \mathrm{d} \rho
$$

4. The stresses $\sigma_{i j}^{(n)}$ are updated through the relation; $\sigma_{i j}=\mathscr{L}_{i j k l} \varepsilon_{k l}^{e}$.

5. Repeat step 1 through 4, until convergence is obtained. Here, convergence is determined based on the displacement and stress fields.

The iterative framework is initiated by using the elastic solution $\left(\gamma^{(\alpha)}=\right.$ 0) in the first iteration. Moreover, the implementation of the framework 
relies on the modifications to the spatial integration suggested by Niordson (2001) and Nielsen and Niordson (2012a). To stabilize the evolution of related field quantities, substeps are introduced as additional points of evaluation in regions with steep gradients between the Gauss points. As the total strain components, $\varepsilon_{i j}$, are unknown between the Gauss points it is necessary to interpolate the strains. In the present paper, it was chosen to interpolate the strain components by assuming a linear variation between the Gauss points. This modification has proven to be particularly important when approaching the rate independent limit $(m \rightarrow 0)$.

\section{Results}

The effective in-plane slip systems of the monazite crystal, derived in Section 3.2 , are oriented non-symmetrically with respect to the external loading causing a non-symmetric in-plane deformation field and thus the entire domain needs to be modelled. The mesh is constructed such that the contact points are located in a finely meshed region, in order to achieve sufficient resolution of the results, while the mesh gradually becomes coarser when moving away from the contact point singularities (see domain in Fig. 4). For the results presented in this study there is a total of 87,120 elements in the entire domain, where approximately 60,000 of the elements are located in the "Fine mesh" region (see Fig. 4). Furthermore, it should be noticed that the rate-dependent model, employed in the current study, cannot predict actual discontinuities but rather rays with a very narrow, but finite, width. 
However, the rays will be denoted as discontinuities in the following.

\subsection{Stress fields}

The stress distribution for the wedge indented monazite crystal is first presented as contour plots in Fig. 5. Details on the stress quantities in the vicinity of the moving contact point singularities are then extracted along different angular paths (see Figs. 7 and 8), and lastly the stresses very near the contact points are presented as stress trajectories (see Figs. 9 and 10). The contour plots are presented in a self-similar coordinate system, $\xi_{i}$, such that the right contact point singularity is always located at the coordinate $\xi_{i}=(1,0)$. For the parameters chosen in this study, the monazite material has been found to have a hardness of $H \approx 5.2 \tau_{0}$, by dividing the sum of vertical reaction forces acting on the indenter by the projected contact area.

In contrast to the effective slip systems investigated by Saito et al. (2012) and Juul et al. (2018) (for FCC, BCC, and HCP crystals), the stress distribution for monazite shows no symmetry with respect to the vertical indentation axis (see Fig. 5). This observation is directly linked to the non-symmetric orientation of the slip systems, with respect to the indenter, and this drives the two contact points to different locations relative to the center axis, $\xi_{3}$. In fact, the ratio of the distance to the two contact points is found to be $a_{r} / a_{l} \approx 1.04$. Thus, the left contact point is located the closest to the $\xi_{3}$-axis which implies that it travels at a lower velocity compared to the right contact point. Upon inspection of the stress contours, in the immediate vicinity of 
the contact points, it is seen that stress rays emanate from these characteristic points similarly to the FCC, BCC and HCP cases. This suggests that an asymptotic solution should exist close to the contact points in line with the predictions of Saito and Kysar (2011) for FCC and BCC crystals. In analogy, the asymptotic solution of the tangential stress distribution is expected to be independent of the distance in the immediate vicinity of the contact point while it is expected to break down further away.

To establish the asymptotic solution numerically, the angular variation of the stresses near the contact points are extracted by employing inverse isoparametric mapping (Murti et al., 1988; Lim et al., 1992) within the finite element mesh (the angular paths are illustrated in Fig. 6). The stress quantities are extracted at four different radii to confirm that the paths are within a region with small radial variations (similar to the observations for FCC and BCC crystals). The stress quantities are presented in Figs. 7 and 8 for the left and right contact point, respectively. Upon inspection of the angular stress variation, at the four different radii, it is seen that the results gradually changes when increasing the radius. However, the region $r<0.25 a$ is considered to be governed by the asymptotic solution due to the relatively small variation. From Figs. 7 and 8 it is also worth to notice that the stress components satisfy the boundary conditions as $\sigma_{13}=\sigma_{33}=0$ at $\psi=0$ (at the free surface) and $\sigma_{13}=0$ at $\psi=-180^{\circ}$ (at the frictionless indenter surface). Comparing the stresses of the left (Fig. 7) and the right (Fig. 8) contact points, it is found that the $\sigma_{13}$ components reaches approximately 
the same maximum magnitude. However, the right contact point reaches a higher level of $\sigma_{11}$, whereas the $\sigma_{33}$ component is similar to the left contact point.

The stress trajectories for different radii are plotted in Figs. 9 and 10 for the left and right contact point, respectively, starting at the star marker which is at the free surface $(\psi=0)$, and moving to the indenter surface $\left(\psi=-180^{\circ}\right)$. Comparing the stress trajectory at the four different radii, it is seen that the characteristics of the trajectories gradually change, and again the two radii closest to the contact point are reasonably similar (inside the region $r<0.25 a$ where the asymptotic solution is assumed valid). For the left contact point (Fig. 9), it is observed that the stress trajectory moves in a counter clockwise direction towards the yield surface, whereafter it stays on the yield surface before it reenters the elastic region and returns to the region in stress space where it started. For the right contact point (Fig. 10), the stress trajectory moves in opposite direction (clockwise direction) towards the yield surface and then remains on the yield surface approaching the vertex. However, before reaching the vertex, the stress state again reenters the elastic region and approaches the starting point. Based on the stress trajectory extracted for both contact points, which only reaches the part of the yield surface related to slip system (2), it is expected to observe a slip rate discontinuity on slip system (2) only. This will be investigated in the following study of slip rates. Also notice that the stress trajectory stays on the yield surface in a small region due to finite width of the ray caused by 
the rate dependent model.

\subsection{Slip rate fields}

The slip rate fields for the wedge indented monazite is first presented as contour plots, to give an overview of the activity of the various slip planes in the vicinity of the moving contact points (see Fig. 11), and secondly as angular plots of the slip rate near the left and right contact points (see Figs. 12 and 13, respectively) to share details on the activity. The main goal is to bring out the discontinuities expected in the slip rate field, demonstrated by Drugan and Rice (1984); Rice (1987); Saito and Kysar (2011); Juul et al. (2018) for FCC and BCC crystals.

Figure 11 displays the normalized slip rate on the two slip systems as well as the total slip rate (the sum $\left.\dot{\gamma}^{\text {(tot) }}=\sum_{A}\left|\dot{\gamma}^{(A)}\right|\right)$. Even though an analytical solution has not been established for monazite, discontinuities are expected in the slip rate field and the first signs of a discontinuity on slip system (2) for both contact points have already revealed themselves when the stress trajectory was investigated. By examining the slip rates, it is seen that the slip intensifies on slip system (1) along a ray emanating from the right contact point along $\psi=-103.21^{\circ}$ (suggesting a kink shear ray), however, the ray never reaches the contact point and the stress trajectory close to the contact point does not reach the yield surface for slip system (1). Thus, this is not a discontinuity related to the asymptotic solution. Instead the very limited activity on slip system (1) is an artifact of the far field conditions. 
When inspecting the activity on slip system (2) it is seen that a discontinuity arises at $\psi=-90^{\circ}$ (forming a glide shear ray), which is evident at both the left and right contact point corresponding to the predictions from the stress trajectory. Furthermore, by comparing the slip rate on slip system (2) at the two contact points it is seen that they are within the same order of magnitude (see Fig. 11a). From the total slip rate in Fig. 11c, the interaction between slip system (1) and (2) is obvious in the right half of the domain (even though the activity on slip system (1) is not related to the asymptotic solution).

The angular variation of the slip rates near the two contact points is investigated closer in the following by using the same inverse isoparametric mapping scheme employed for the corresponding plots of the stress quantities (see the angular path in Fig. 6). The slip activity indicates the location of the sector boundaries as it divides regions into either elastic or plastic regions. The slip rates are presented in Figs. 12 and 13 for the left and right contact point, respectively. Inspection of the left contact point in Fig. 12 reveals two spikes in the slip rates, however, the spike on slip system (1) is of negligible magnitude as was also observed in Fig. 11a, and hence it should not be interpreted as evidence of a discontinuity. On slip system (2), the predicted discontinuity is clearly seen at $\psi=-90^{\circ}$, and this discontinuity maintains its orientation at the different radii of data extraction indicating that the asymptotic solution stretches far into the domain even though its theoretical validity is expected only to hold near the contact point.

For the right contact point (in Fig. 13), the same clear spike is seen for 
slip system (2) at $\psi=-90^{\circ}$, as expected. For slip system (1) a spike is again observed, but its magnitude is very low and is not oriented at the expected angle. This also supports the previous conclusions, stating that a discontinuity on slip system (1) emanating from the contact point does not exist. At the largest radius of data extraction, the activity on slip system (1) starts to increase, however, the spike is still not located at the expected angle and is not related to the asymptotic solution which is in focus here.

\section{Concluding remarks}

Numerical simulations of 2D wedge indentation in an elastic, perfectly plastic monazite single crystal have been carried out. The main focus in the numerical analysis was to investigate the material behaviour in the immediate vicinity of the moving contact point singularities. The variations in the deformation, stress, and slip rate fields lead to the following key findings:

- Indentation in the monazite crystal reveals a non-symmetric deformation field. This is seen by the contact length and velocity of the left and right contact points being different. For the monazite crystal, with the given parameters, the right contact point travels faster than the left contact point, with a constant ratio of $a_{r} / a_{l} \approx 1.04$, between the contact lengths.

- The stress distribution in the vicinity of the contact point singularities exhibits a non-symmetric field around the indenter. By investigating 
the angular variations in the stress field it is seen that, in the immediate vicinity of the contact points, an asymptotic field exists independent of the distance to the singularity. This is in-line with the findings of Saito and Kysar (2011) for FCC and BCC crystals. The stress trajectory for the monazite shows regions where the path reaches the yield surface, implying the existence of two elastic sectors.

- The numerical simulation reveals the existence of discontinuities in the slip rate, similar to those predicted by Saito and Kysar (2011) for FCC and BCC crystals. For the monazite crystal, a discontinuity parallel to slip system (2), causing glide shear, was predicted at both contact points with the activity on slip system (2) being on the same order of magnitude. Slip system (1) was essentially found to be inactive near the contact points and did not indicate any discontinuities.

- For the parameters chosen in this study, the monazite material has been found to have a hardness of $H \approx 5.2 \tau_{0}$.

Based on numerical calculations, a sector structure for the monazite material, similar to the analytical structure predicted by Saito and Kysar (2011) for FCC and BCC, is suggested in Fig. 14. Here, the material behaviour is divided into two elastic sectors around each contact point. 
444

445

446

447

448

449

450

451

\section{Acknowledgments}

The work is financially supported by The Danish Council for Independent Research in the projects "New Advances in Steady-State Engineering Techniques", grant DFF-4184-00122 and "Higher Order Theories in Solid Mechanics", grant 11-105098. JWK gratefully acknowledges support from NSF DMR-1310503. Associate Professor Chris Valentin Nielsen, DTU Mechanical Engineering, is acknowledged for his parallelization of the employed skyline solver module (Nielsen et al., 2012). 


\section{References}

Bastawros, A.-F., Kim, K.-S., 1998. Experimental analysis of deformation induced microstructure near a crack tip in a hardened copper crystal. MRS Proceedings 539 .

Crone, W., Shield, T., 2001. Experimental study of the deformation near a notch tip in copper and copperberyllium single crystals. Journal of the Mechanics and Physics of Solids 49 (12), 2819 - 2838.

Crone, W., Shield, T., Creuziger, A., Henneman, B., 2004. Orientation dependence of the plastic slip near notches in ductile $\{F C C\}$ single crystals. Journal of the Mechanics and Physics of Solids 52 (1), 85 - 112.

Dahlberg, C. F. O., Saito, Y., Öztop, M. S., Kysar, J. W., 2017. Geometrically necessary dislocation density measurements at a grain boundary due to wedge indentation into an aluminum bicrystal. Journal of the Mechanics and Physics of Solids 105, 131-149.

Dahlberg, C. F. O., Saito, Y., ztop, M. S., Kysar, J. W., 2014. Geometrically necessary dislocation density measurements associated with different angles of indentations. Int. J. Plas. 54, 81-95.

Davis, J. B., Hay, R. S., Marshall, D. B., Morgan, P. E. D., Sayir, A., 2003. Influence of interfacial roughness on fiber sliding in oxide composites with la-monazite interphases. Journal of the American Ceramic Society 86 (2), 305-316. 
Donnay, J. D. H., May 1943. Rules for the conventional orientation of crystals. The American Mineralogist 28 (5), 313-328.

Drugan, W. J., 1986. A more direct and general-analysis of moving strong discontinuity surfaces in quasi-statically deforming elasticplastic solids. J. Appl. Mech. 53, 224-226.

Drugan, W. J., 2001. Asymptotic solutions for tensile crack tip fields without kink-type shear bands in elastic-ideally plastic single crystals. J. Mech. Phys. Solids 49, 2155-2176.

Drugan, W. J., Rice, J. R., 1984. Restrictions on quasi-statically moving surfaces of strong discontinuity in elasticplastic solids. In: Dvorak, G.J., Shield, R.T. (Eds.), Mechanics of Material Behavior (The D.C. Drucker Anniversary Volume). Elsevier, 59-73.

Drugan, W. J., Rice, J. R., Sham, T. L., 1982. Asymptotic analysis of growing plane-strain tensile cracks in elastic ideally plastic solids. J. Mech. Phys. Solids 30, 447-473.

Feng, J., Xiao, B., Zhou, R., Pan, W., 2013. Anisotropy in elasticity and thermal conductivity of monazite-type repo4 (re = la, ce, nd, sm, eu and gd) from first-principles calculations. Acta Materialia 61 (19), 7364-7383.

Grechanovsky, A. E., Eremin, N. N., Urusov, V. S., 2013. Radiation resistance of lapo4 (monazite structure) and ybpo4 (zircon structure) from data of computer simulation. Physics of the Solid State 55 (9), 1929-1935. 
Hay, R. S., 2005. Twindislocation interaction in monazite (monoclinic lapo4). Philosophical Magazine 85 (2-3), 373-386.

Hay, R. S., 2008. Monazite (monoclinic $\mathrm{LaPO}_{4}$ ) slip systems at room temperature. Phil. Mag. 88, 4243-4270.

Hay, R. S., Marshall, D. B., 2003. Deformation twinning in monazite. Acta Mat. 51, 5235-5254.

Hirth, J. P., Lothe, J., 1968. Theory of dislocations. McGraw-Hill,.

Hutchinson, J. W., 1976. Bounds and self-consistent estimates for creep of polycrystalline materials. Proc. R. Soc. Lond. A 348, 101-127.

Juul, K. J., Nielsen, K. L., Niordson, C. F., 2017. Steady-state crack growth in single crystals under mode i loading. J. Mech. Phys. Solids 101, 209-222.

Juul, K. J., Niordson, C. F., Nielsen, K. L., Kysar, J. W., 2018. A novel numerical framework for self-similarity in plasticity: Wedge indentation in single crystals. Journal of the Mechanics and Physics of Solids 112, 667684.

Kysar, J. W., 2000. Directional dependence of fracture in copper/sapphire bicrystal. Acta Materialia 48 (13), 3509-3524.

Kysar, J. W., 2001a. Continuum simulations of directional dependence of crack growth along a copper/sapphire bicrystal interface. Part I: Exper- 
iments and crystal plasticity background. Journal of the Mechanics and Physics of Solids 49 (5), 1099-1128.

Kysar, J. W., 2001b. Continuum simulations of directional dependence of crack growth along a copper/sapphire bicrystal interface. Part II: Crack tip stress/deformation analysis. Journal of the Mechanics and Physics of Solids 49 (5), 1129-1153.

Kysar, J. W., Briant, C. L., 2002. Crack tip deformation fields in ductile single crystals. Acta Materialia 50 (9), 2367-2380.

Kysar, J. W., Gan, Y. X., Mendez-Arzuza, G., 2005. Cylindrical void in a rigid-ideally plastic single crystal. Part I: Anisotropic slip line theory solution for face-centered cubic crystals. Int. J. Plas. 21, 1481-1520.

Kysar, J. W., Saito, Y., Öztop, M. S., Lee, D., Huh, W. T., 2010. Experimental lower bounds on geometrically necessary dislocation density. International Journal of Plasticity 26 (8), 1097-1123.

Lim, I. L., Johnston, I. W., Choi, S. K., Murti, V., 1992. An improved numerical inverse isoparametric mapping technique for $2 \mathrm{~d}$ mesh rezoning. Eng.Frac. Mech. 41, 417-435.

Matthies, S., Wenk, H.-R., Aug 2009. Transformations for monoclinic crystal symmetry in texture analysis. Journal of Applied Crystallography 42 (4), $564-571$. 
Mesarovic, S. D., Kysar, J. W., 1996. Continuum aspects of directionally dependent cracking of an interface between copper and alumina crystals. Mechanics of Materials 23 (4), 271-286.

Murti, V., Wang, Y., Valliappan, S., 1988. Numerical inverse isoparametric mapping in 3d fem. Computers and Structures 29, 611-622.

Nielsen, C. V., Zhang, W., Alves, L. M., Bay, N., Martins, P. A. F., 2012. Modeling of Thermo-Electro-Mechanical Manufacturing Processes with Applications in Metal Forming and Resistance Welding, First Edition. Springer.

Nielsen, K. L., Niordson, C. F., 2012a. Rate sensitivity of mixed mode interface toughness of dissimilar metallic materials: Studied at steady state. Int. J. Solids Struct. 49, 576-583.

Niordson, C. F., 2001. Analysis of steady-state ductile crack growth along a laser weld. Int. J. Frac. 111, 53-69.

Niordson, C. F., Kysar, J. W., 2014. Computational strain gradient crystal plasticity. J. Mech. Phys. Solids 62, 31-47.

Rice, J. R., 1987. Tensile crack tip fields in elastic-ideally plastic crystals. Mech. Mater. 6, 317-335.

Ruggles-Wrenn, M. B., Yeleser, T., Fair, G. E., Davis, J. B., 2009. Effects of steam environment on creep behavior of Nextel $^{\mathrm{TM}} 610 /$ monazite/alumina composite at $1,100^{\circ} \mathrm{C}$. Applied Composite Materials 16 (6), 379-392. 
Saito, Y., Kysar, J. W., 2011. Wedge indentation in to elastic-plastic single crystals, 1: Asymptotic fields for nearly-flat wedge. Int. J. Plas. 27, 16401657.

Saito, Y., Oztop, M. S., Kysar, J. W., 2012. Wedge indentation in to elasticplastic single crystals, 2: Simulations for face-centered cubic crystals. Int. J. Plas. 28, 70-87.

Sarac, A., Kysar, J. W., 2018. Experimental validation of plastic constitutive hardening relationship based upon the direction of the net burgers density vector. Journal of the Mechanics and Physics of Solids 111, 358-374.

Sarac, A., Oztop, M. S., Dahlberg, C. F. O., Kysar, J. W., 2016. Spatial distribution of the net Burgers vector density in a deformed single crystal. International Journal of Plasticity 85, 110 - 129. 


\section{List of Figures}

1 Wedge indentation in a rate-sensitive single crystal. The developed numerical scheme, exploiting the self-similar properties, is applied inside the elastic-plastic domain, whereas the material is treated as linear elastic outside this domain. . . . . 35

2 Monazite crystal structure showing (a) the monoclinic crystal system, (b) the $2 \mathrm{D}$ representation of the slip in the $x_{1} x_{3}$-plane, (c) the crystallographic orientation of the in-plane slip systems relative to the indenter, and (d)-(f) represent the in-plane slip systems. . . . . . . . . . . . . . . . 36

3 Outer envelope (dashed line) and inner envelope (full line) that make-up the yield surface of monazite, depicted in normalized stress space $\left(\left(\sigma_{11}-\sigma_{33}\right) /\left(2 \tau_{0}\right), \sigma_{13} / \tau_{0}\right)$. Each set of parallel line segments belong to the slip system indicated by the number in parentheses and is oriented the angle $2 \theta^{(A)}$ (see Table 3 ) with respect to the $\left(\sigma_{11}-\sigma_{33}\right) / 2 \tau_{0}$-axis. Coordinates of the vertices A - G (the intersect of the line segments) are listed in Table 4.37

4 Domain used for numerical simulations. The dashed arrows indicate the direction of gradually increasing element size. The boundary of the domain is sufficiently far away from the contact point to have negligible influence on the results (the far boundary is clamped).... . . . . . . . . . . . 38

5 Stress distribution, around the moving contact points in wedge indented monazite, for the components; (a) $\sigma_{11} / \tau_{0}$, (b) $\sigma_{22} / \tau_{0}$, and (c) $\sigma_{13} / \tau_{0}$. The contours are plotted in the self-similar coordinate system; $\xi_{i}=x_{i} / a_{r}$, where $a_{r} / a_{l} \approx 1.04$. . . . . . 39

6 Wedge indentation into a monazite single crystal showing left and right contact points and the associated angular paths. . . 40

7 Angular stress distribution for monazite around the left contact point at a radius of; (a) $r=0.15 a_{l}$, (b) $r=0.25 a_{l}$, (c) $r=0.35 a_{l}$, and (d) $r=0.45 a_{l}$ away from the contact point. . . 41

8 Angular stress distribution for monazite around the right contact point at a radius of; (a) $r=0.15 a_{r}$, (b) $r=0.25 a_{r}$, (c) $r=0.35 a_{r}$, and (d) $r=0.45 a_{r}$ away from the contact point.

$9 \quad$ Stress trajectory for monazite around the left contact point at a radius of; (a) $r=0.15 a_{l}$, (b) $r=0.25 a_{l}$, (c) $r=0.35 a_{l}$, and (d) $r=0.45 a_{l}$ away from the contact point. . . . . . . . . 
10 Stress trajectory for monazite around the right contact point at a radius of; (a) $r=0.15 a_{r}$, (b) $r=0.25 a_{r}$, (c) $r=0.35 a_{r}$, and (d) $r=0.45 a_{r}$ away from the contact point. . . . . . . . . 44

11 Slip rate, around the moving contact points in wedge indented monazite, for the slip systems; (a) $\dot{\gamma}^{(1)} / \dot{c}$, (b) $\dot{\gamma}^{(2)} / \dot{c}$, and (c) $\dot{\gamma}^{\text {(tot) }} / \dot{c}$. The contours are plotted in the self-similar coordinate system; $\xi_{i}=x_{i} / a_{r}$, where $a_{r} / a_{l} \approx 1.04$. . . . . . . . . . . 45

12 Angular slip rate distribution for monazite around the left contact point at a radius of; (a) $r=0.15 a_{l}$, (b) $r=0.25 a_{l}$, (c) $r=0.35 a_{l}$, and (d) $r=0.45 a_{l}$ away from the contact point. The dashed lines indicates the potential location of the slip rate discontinuity on slip system $(1)\left(-76.79^{\circ}\right)$ and slip

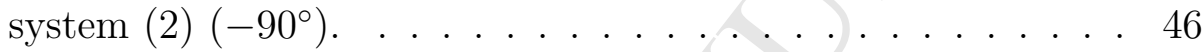

13 Angular slip rate distribution for monazite around the right contact point at a radius of; (a) $r=0.15 a_{r}$, (b) $r=0.25 a_{r}$, (c) $r=0.35 a_{r}$, and (d) $r=0.45 a_{r}$ away from the contact point. The dashed lines indicates the potential location of the slip rate discontinuity on slip system $(1)\left(-103.21^{\circ}\right)$ and slip

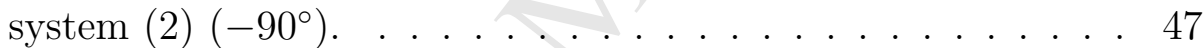

14 Numerically predicted sector structure in the vicinity of contact point singularities governed by an asymptotic solution. . . 48 


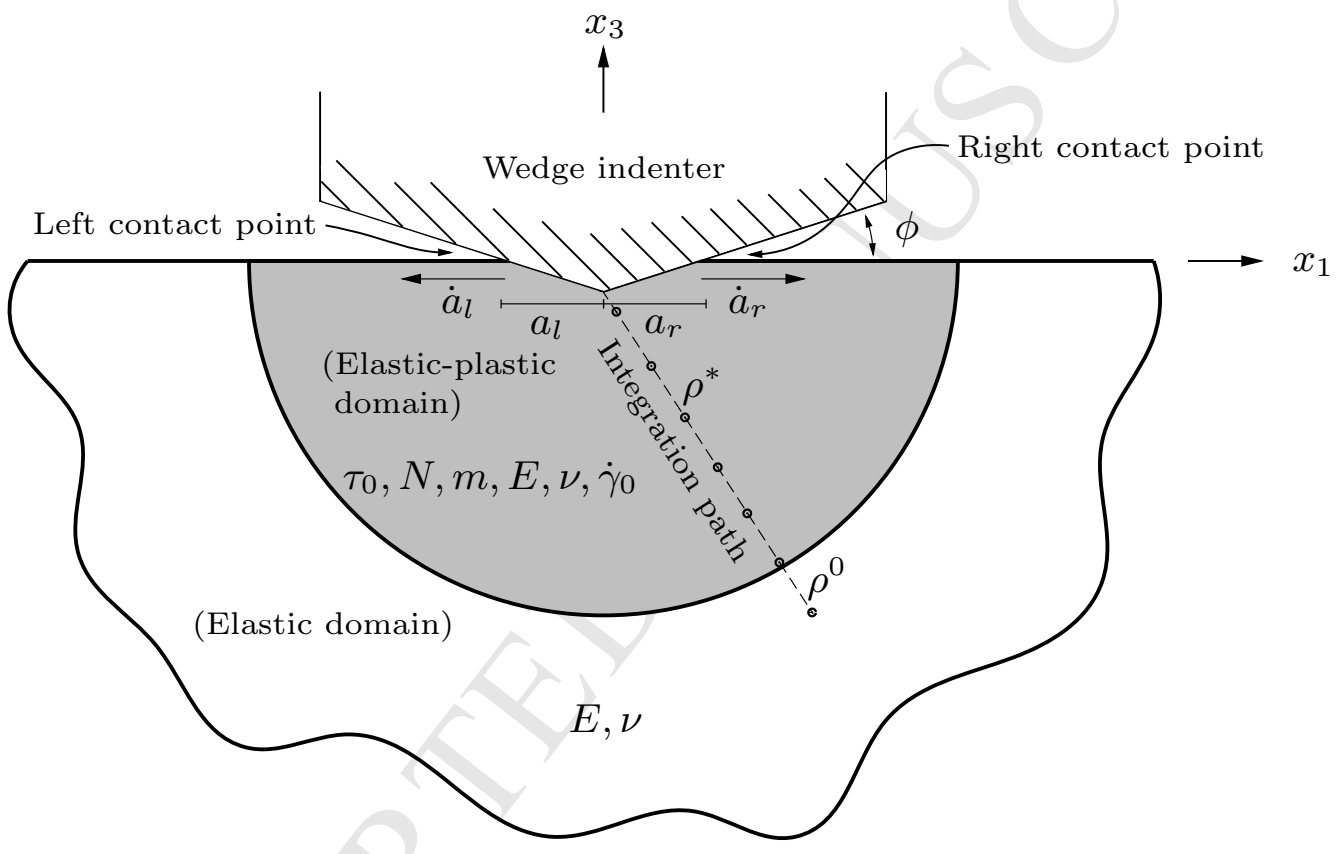

Figure 1: Wedge indentation in a rate-sensitive single crystal. The developed numerical scheme, exploiting the self-similar properties, is applied inside the elasticplastic domain, whereas the material is treated as linear elastic outside this domain. 
(a)

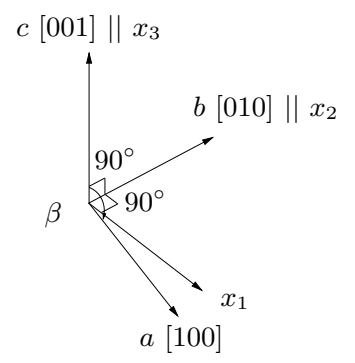

(d)

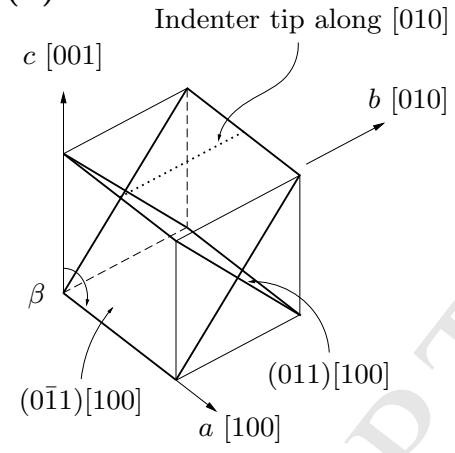

(b)

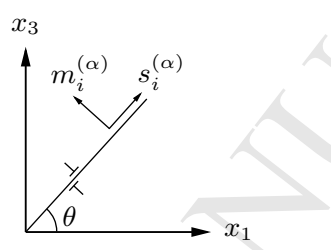

(e)

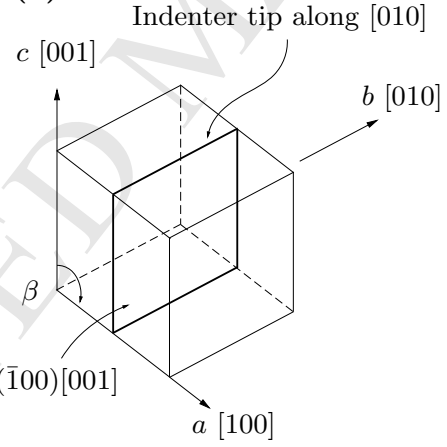

(c)

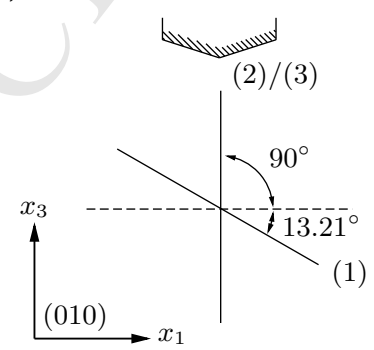

(f)

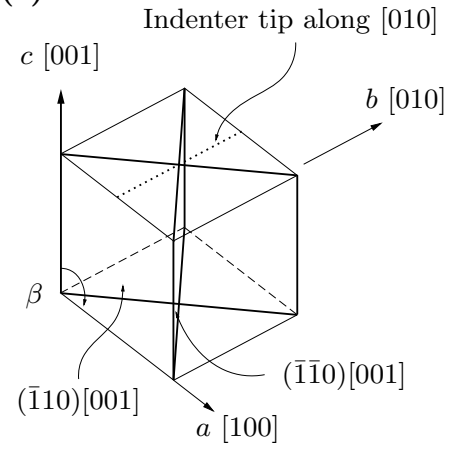

Figure 2: Monazite crystal structure showing (a) the monoclinic crystal system, (b) the 2D representation of the slip in the $x_{1} x_{3}$-plane, (c) the crystallographic orientation of the in-plane slip systems relative to the indenter, and (d)-(f) represent the in-plane slip systems. 


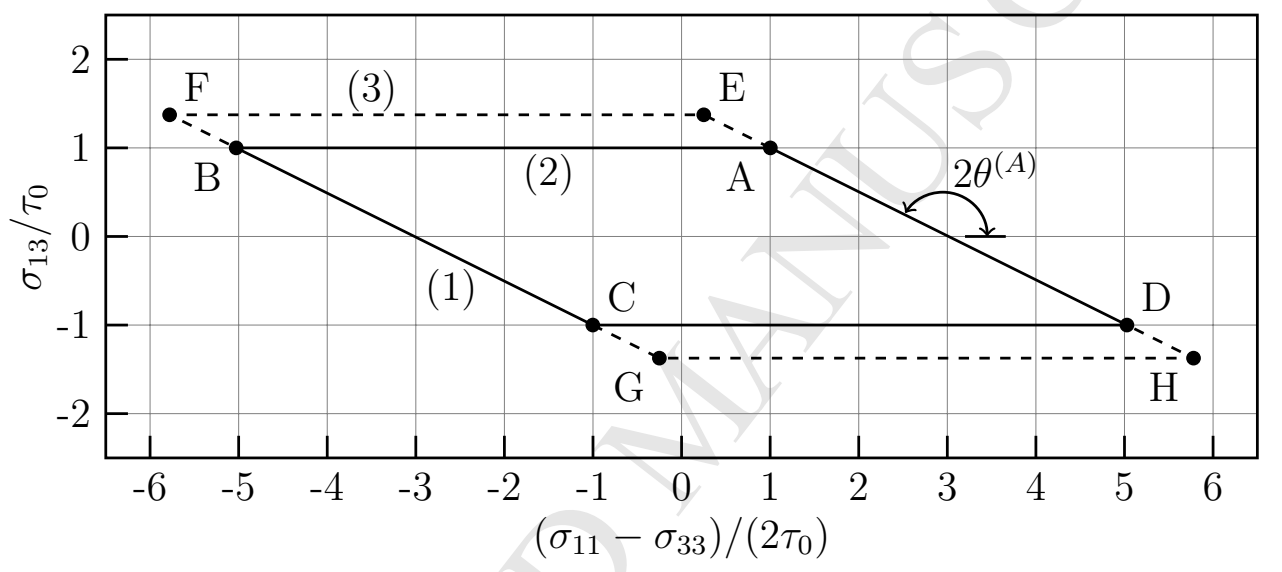

Figure 3: Outer envelope (dashed line) and inner envelope (full line) that make-up the yield surface of monazite, depicted in normalized stress space $\left(\left(\sigma_{11}-\sigma_{33}\right) /\left(2 \tau_{0}\right)\right.$, $\left.\sigma_{13} / \tau_{0}\right)$. Each set of parallel line segments belong to the slip system indicated by the number in parentheses and is oriented the angle $2 \theta^{(A)}$ (see Table 3) with respect to the $\left(\sigma_{11}-\sigma_{33}\right) / 2 \tau_{0}$-axis. Coordinates of the vertices $\mathrm{A}-\mathrm{G}$ (the intersect of the line segments) are listed in Table 4. 


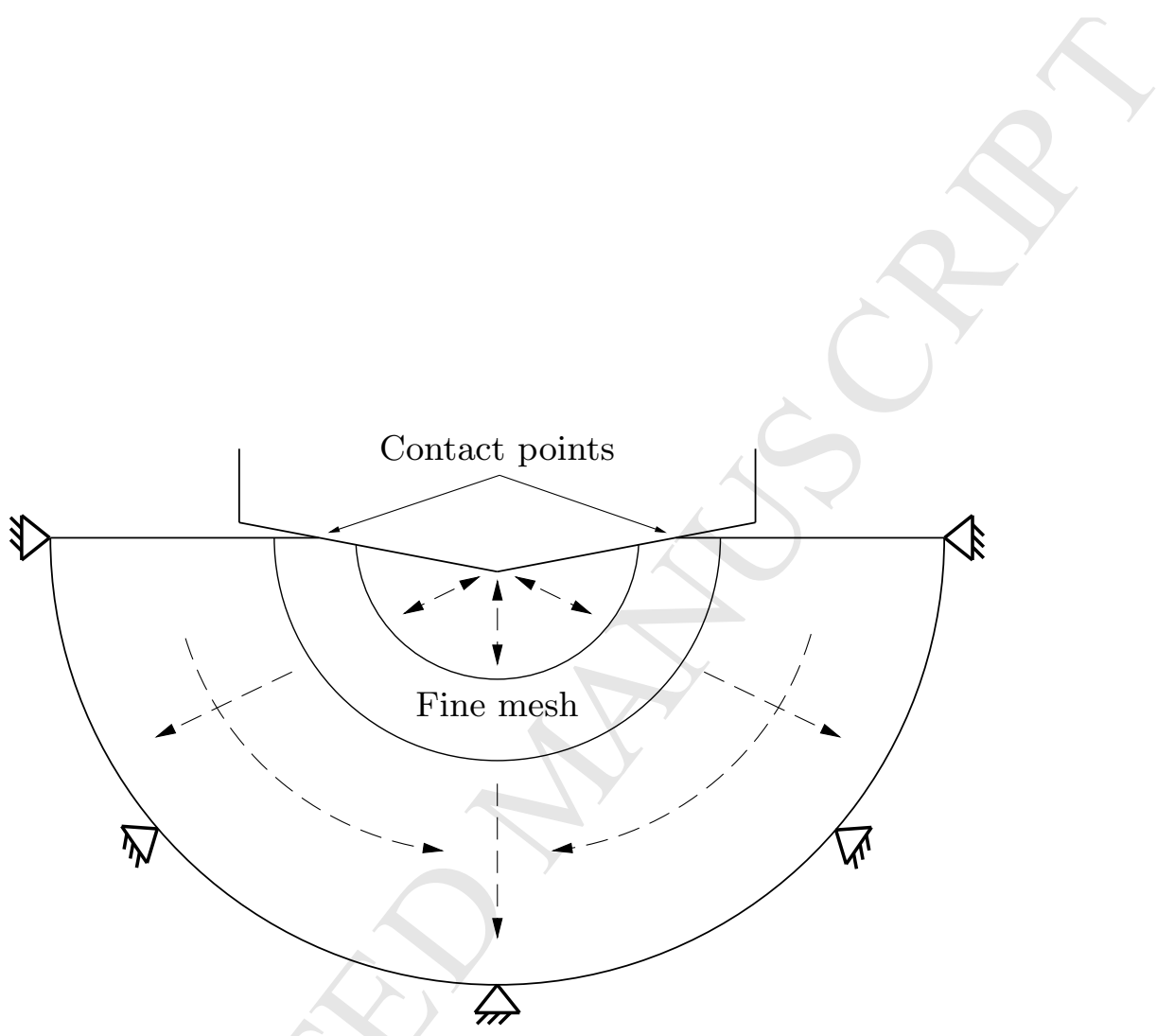

Figure 4: Domain used for numerical simulations. The dashed arrows indicate the direction of gradually increasing element size. The boundary of the domain is sufficiently far away from the contact point to have negligible influence on the results (the far boundary is clamped). 


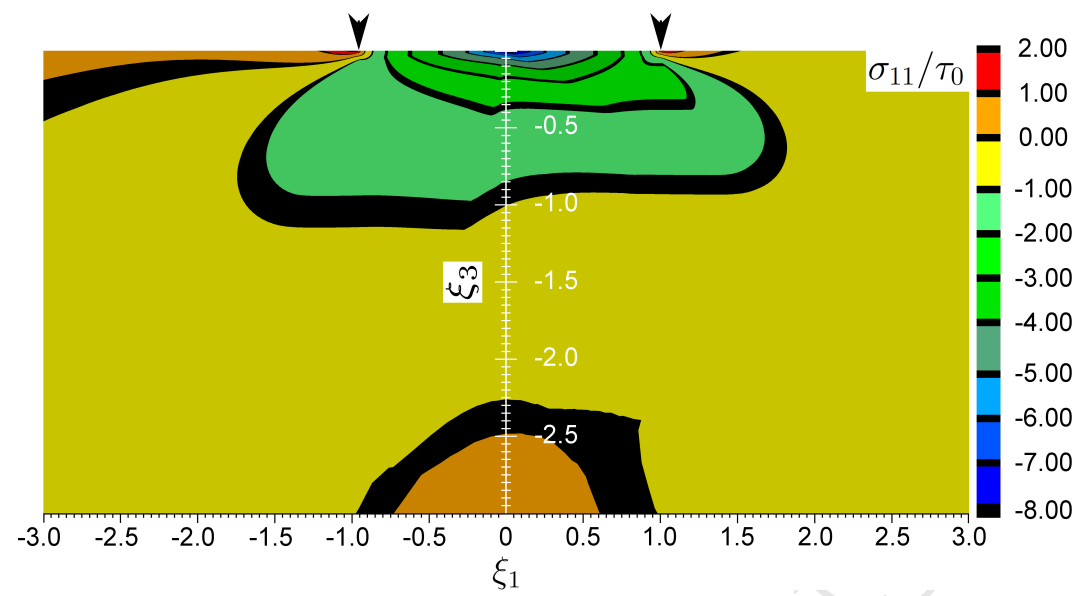

(a)

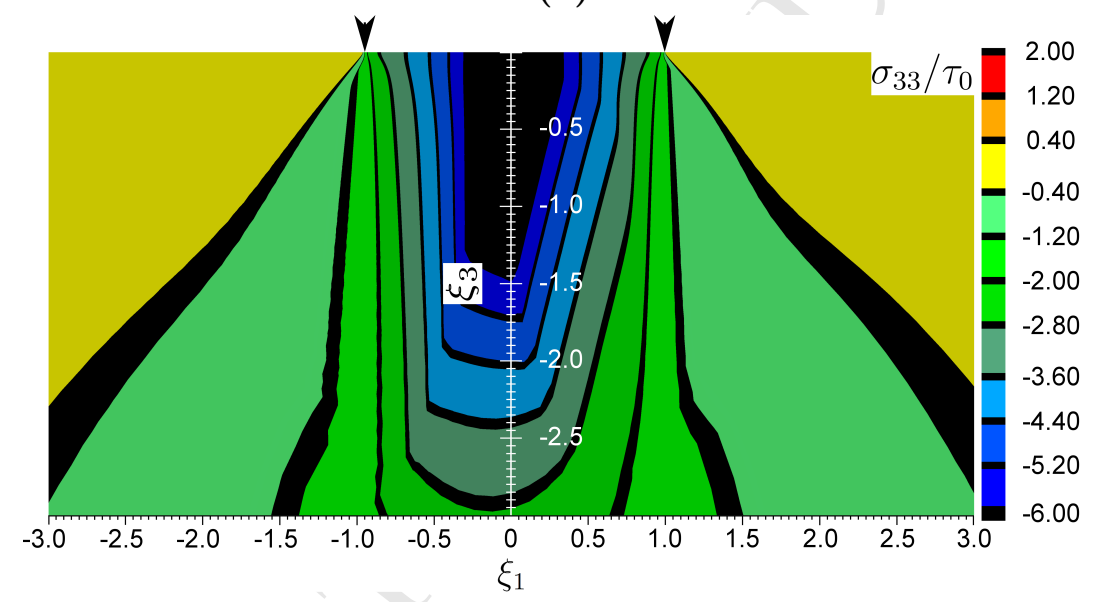

(b)

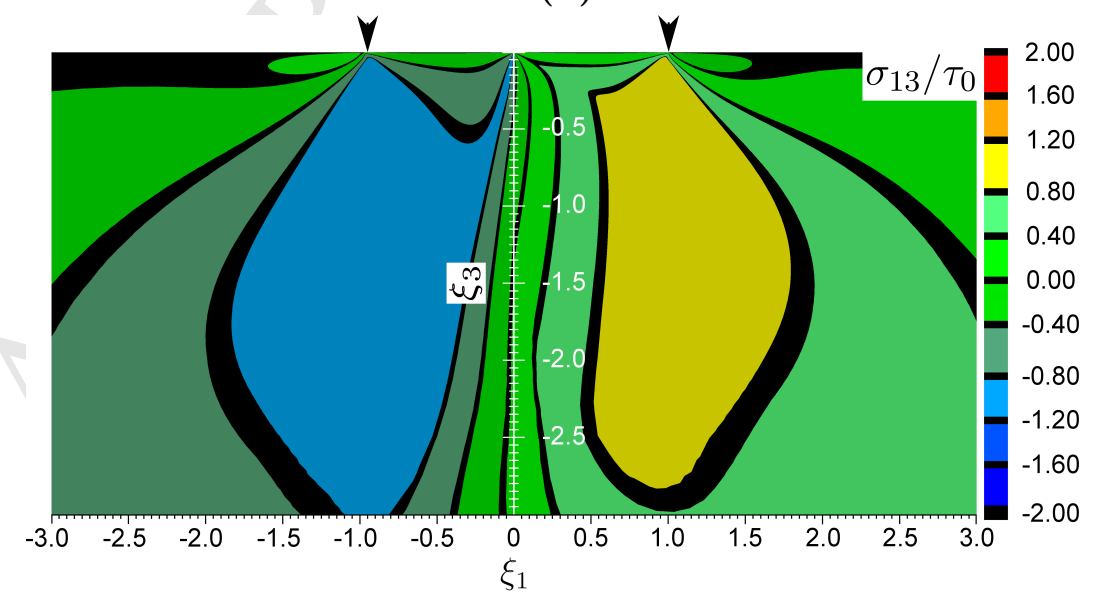

(c)

Figure 5: Stress distribution, around the moving contact points in wedge indented monazite, for the components; (a) $\sigma_{11} / \tau_{0}$, (b) $\sigma_{22} / \tau_{0}$, and (c) $\sigma_{13} / \tau_{0}$. The contours are plotted in the self-similar coordinate system; $\xi_{i}=x_{i} / a_{r}$, where $a_{r} / a_{l} \approx 1.04$. 


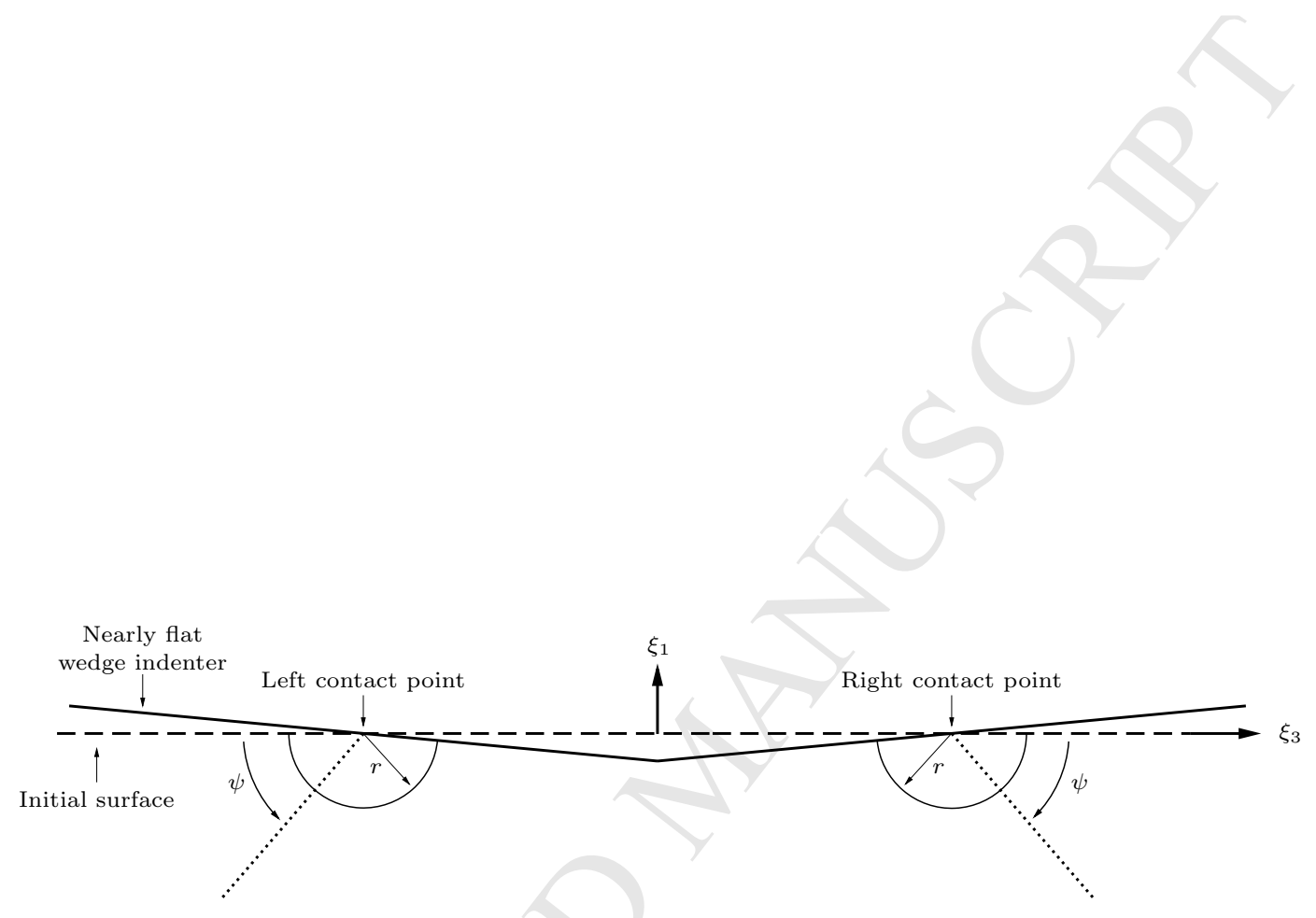

Figure 6: Wedge indentation into a monazite single crystal showing left and right contact points and the associated angular paths. 


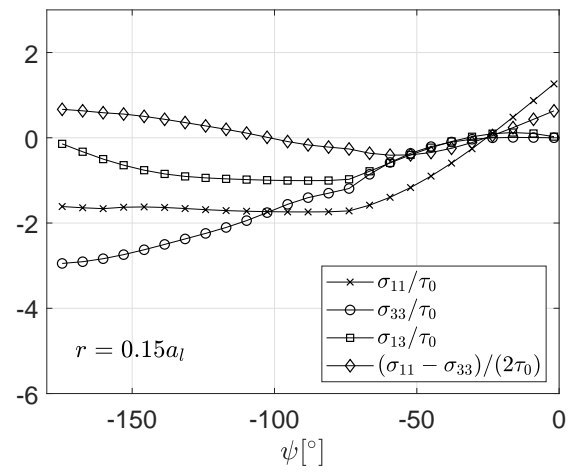

(a)

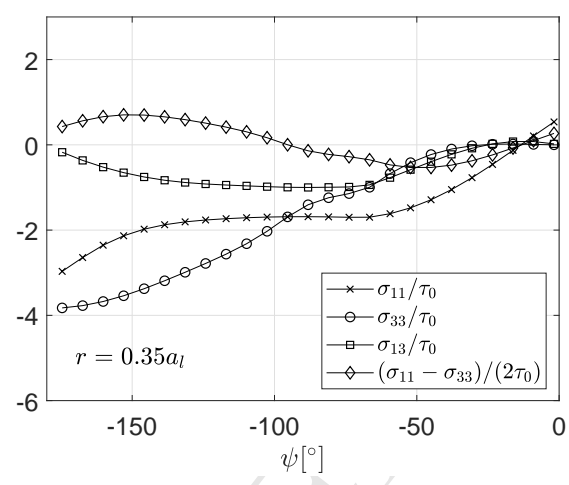

(c)

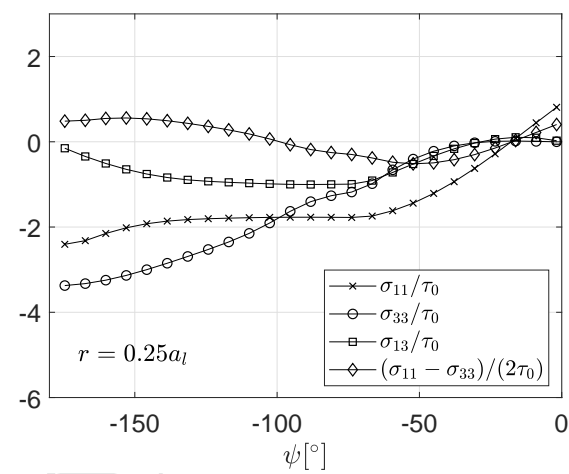

(b)

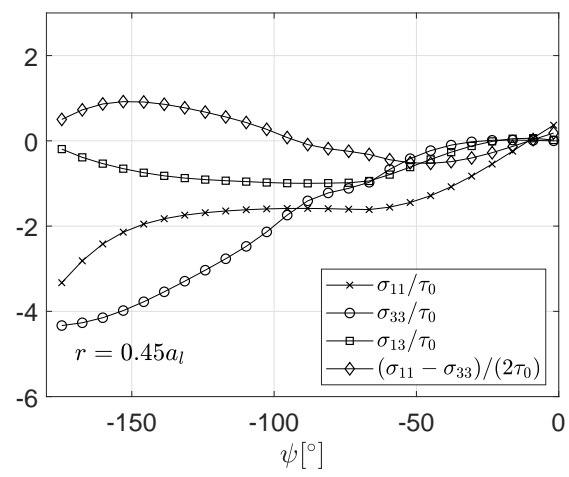

(d)

Figure 7: Angular stress distribution for monazite around the left contact point at a radius of; (a) $r=0.15 a_{l}$, (b) $r=0.25 a_{l}$, (c) $r=0.35 a_{l}$, and (d) $r=0.45 a_{l}$ away from the contact point. 


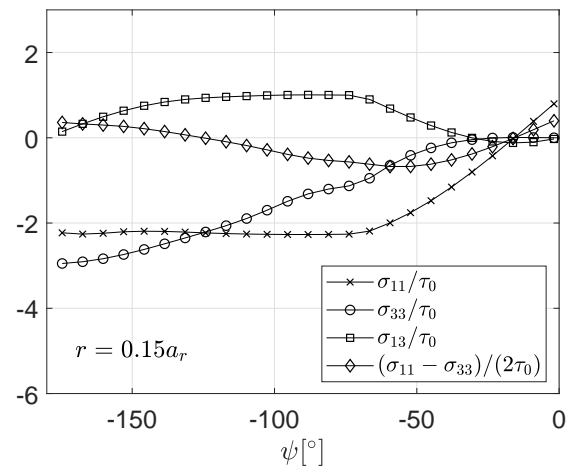

(a)

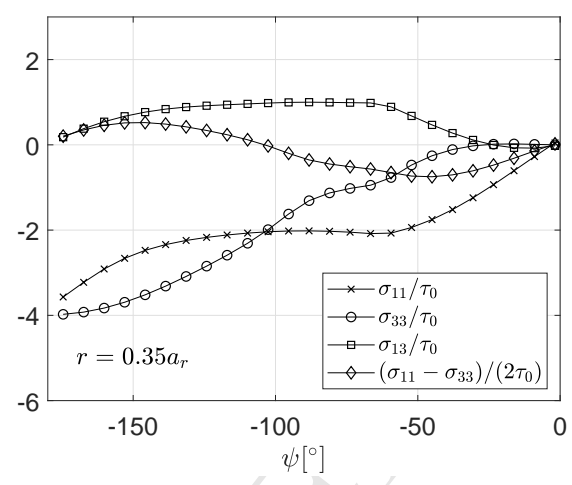

(c)

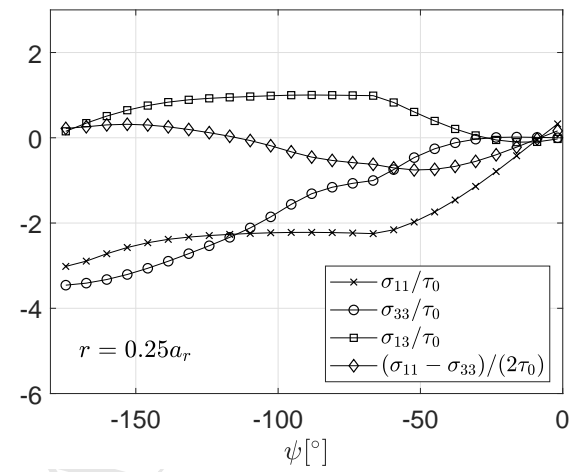

(b)

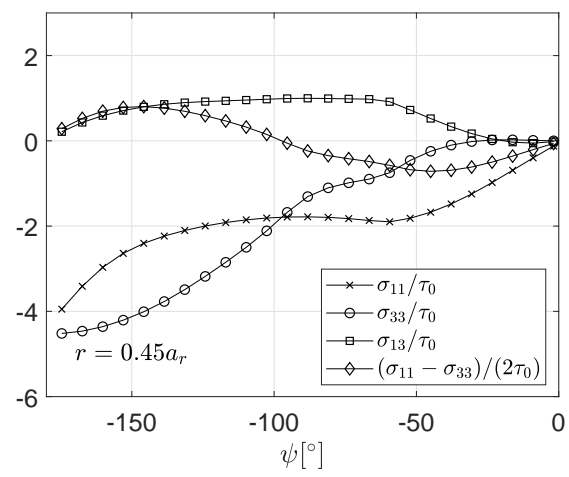

(d)

Figure 8: Angular stress distribution for monazite around the right contact point at a radius of; (a) $r=0.15 a_{r}$, (b) $r=0.25 a_{r}$, (c) $r=0.35 a_{r}$, and (d) $r=0.45 a_{r}$ away from the contact point. 


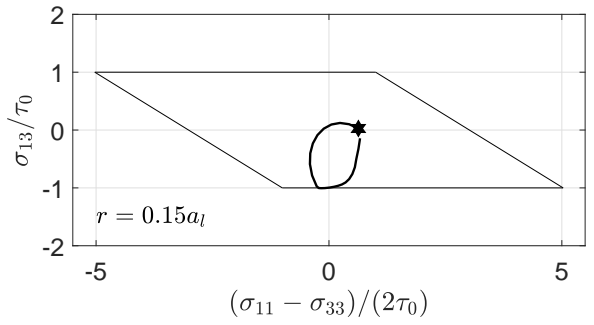

(a)

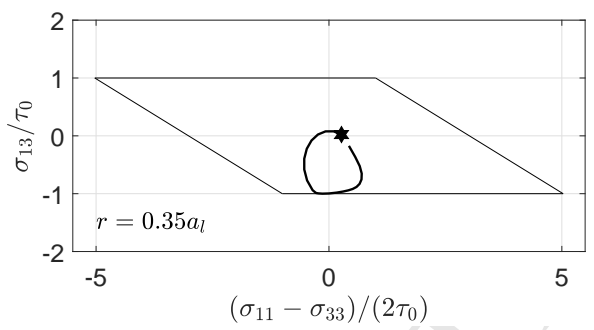

(c)

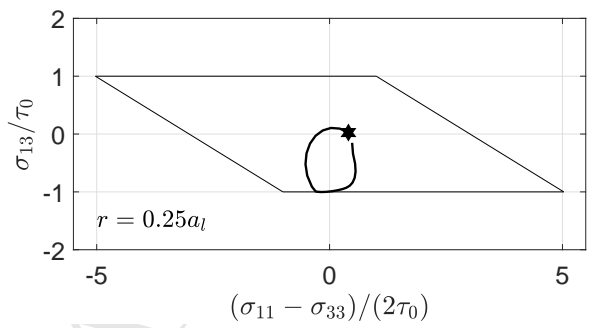

(b)

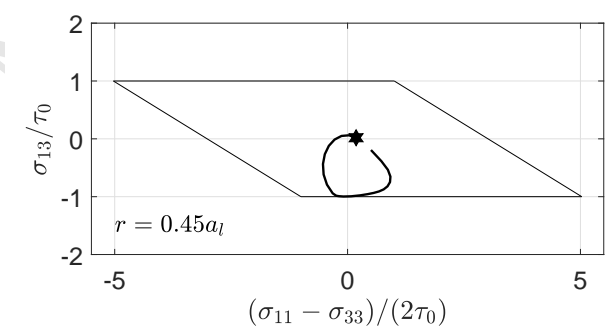

(d)

Figure 9: Stress trajectory for monazite around the left contact point at a radius of; (a) $r=0.15 a_{l}$, (b) $r=0.25 a_{l}$, (c) $r=0.35 a_{l}$, and (d) $r=0.45 a_{l}$ away from the contact point. 


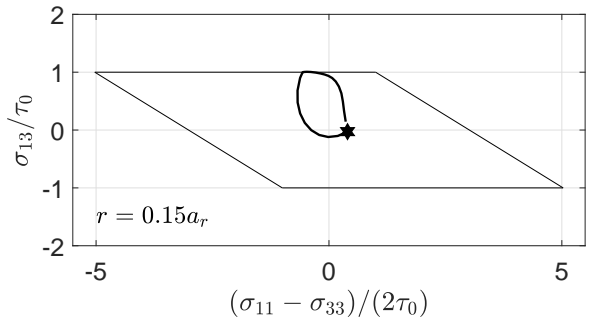

(a)

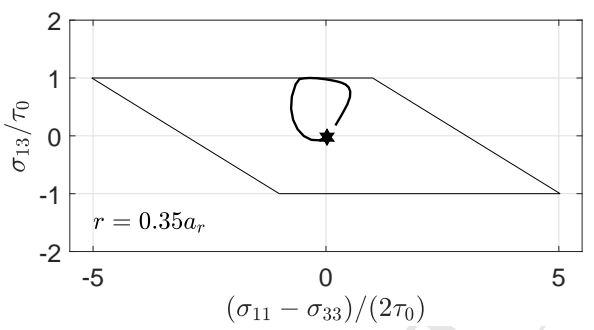

(c)

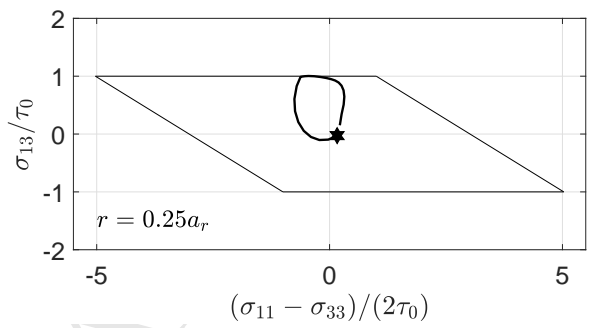

(b)

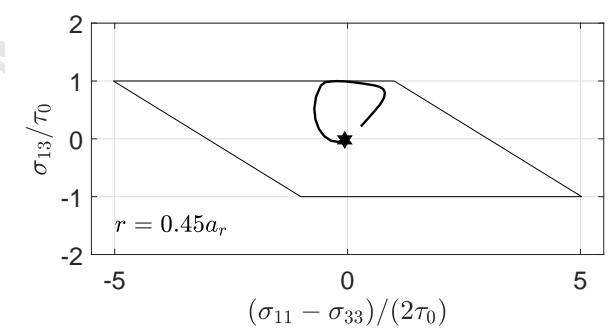

(d)

Figure 10: Stress trajectory for monazite around the right contact point at a radius of; (a) $r=0.15 a_{r}$, (b) $r=0.25 a_{r}$, (c) $r=0.35 a_{r}$, and (d) $r=0.45 a_{r}$ away from the contact point. 


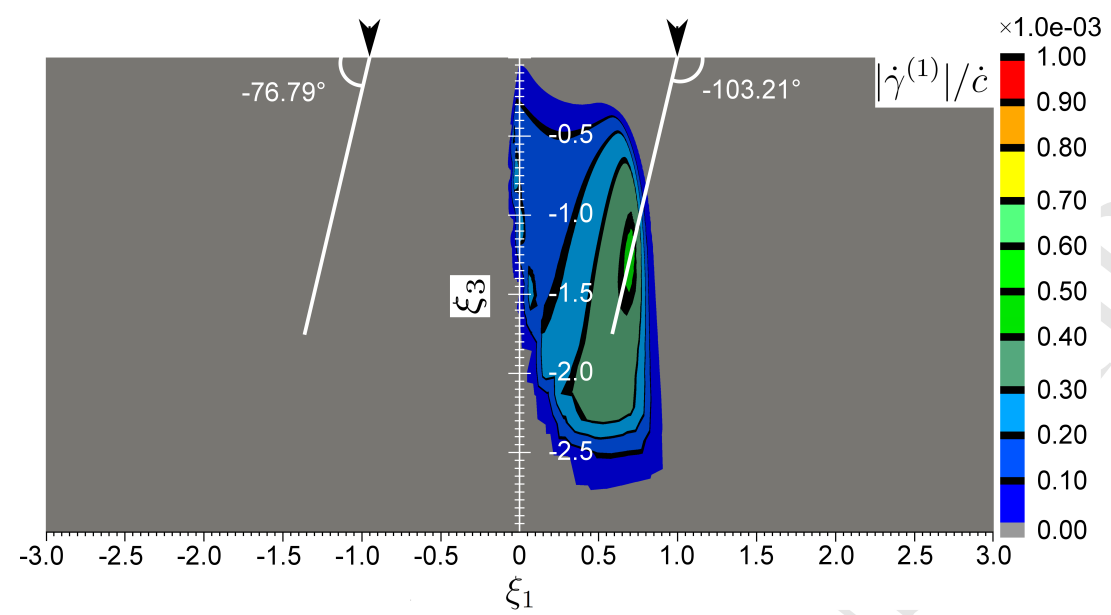

(a)

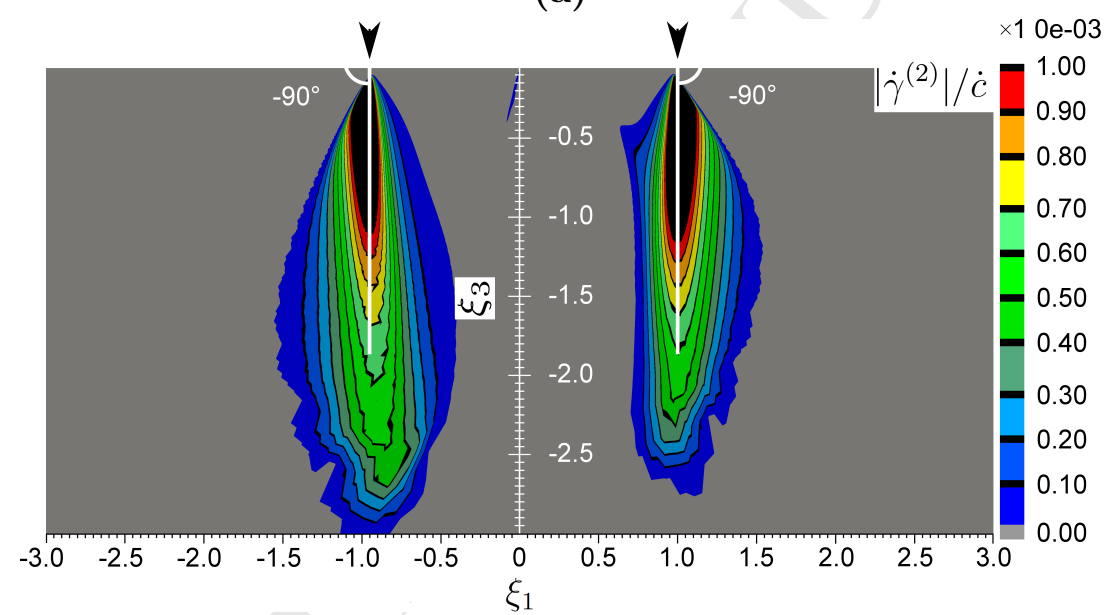

(b)

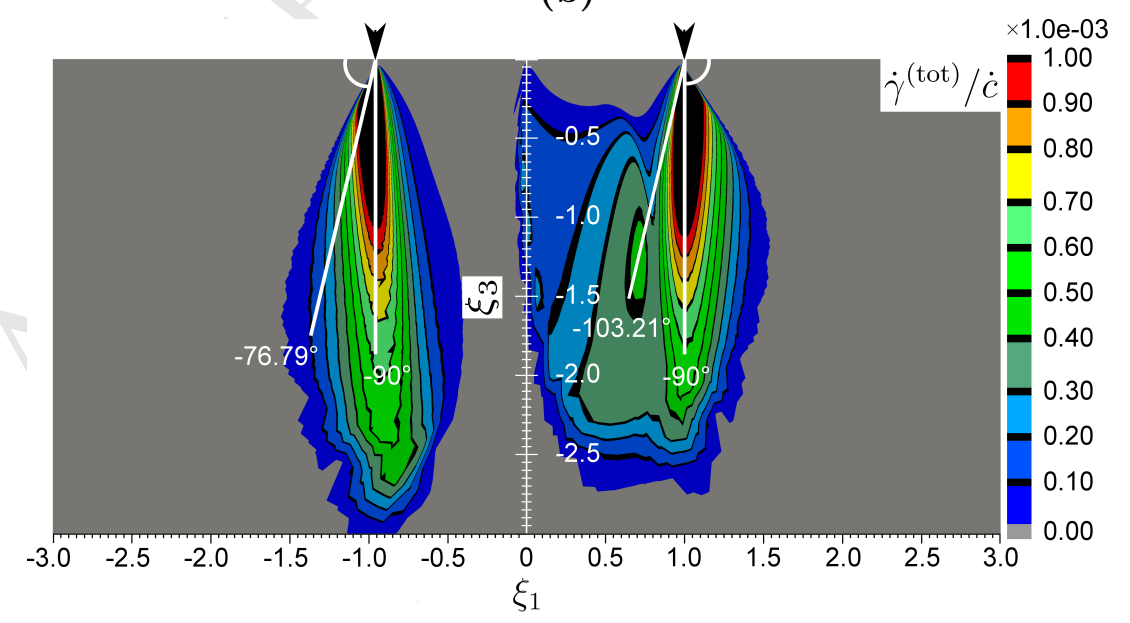

45

Figure 11: Slip rate, around the moving contact points in wedge indented monazite, for the slip systems; (a) $\dot{\gamma}^{(1)} / \dot{c}$, (b) $\dot{\gamma}^{(2)} / \dot{c}$, and (c) $\dot{\gamma}^{\text {(tot) }} / \dot{c}$. The contours are plotted in the self-similar coordinate system; $\xi_{i}=x_{i} / a_{r}$, where $a_{r} / a_{l} \approx 1.04$. 


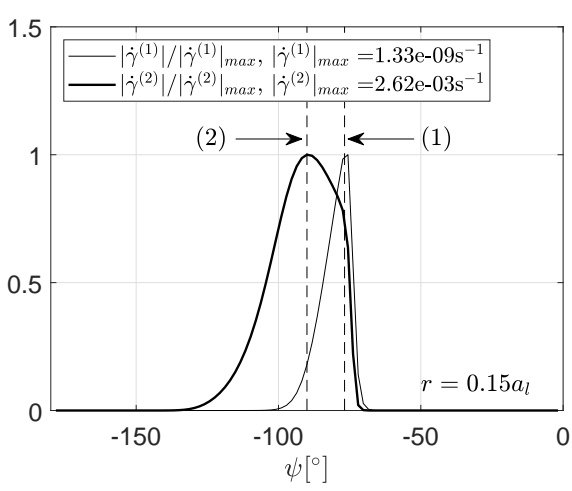

(a)

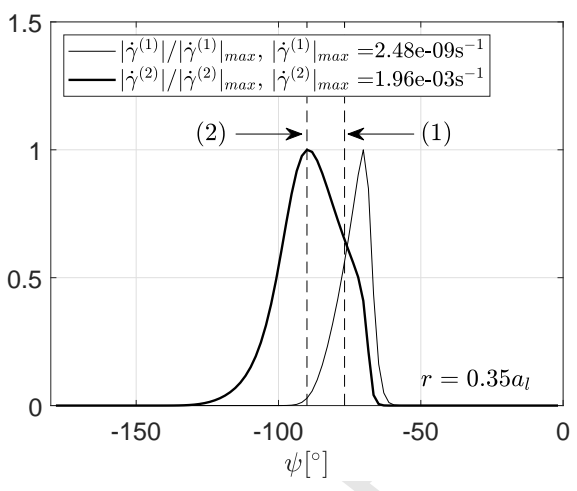

(c)

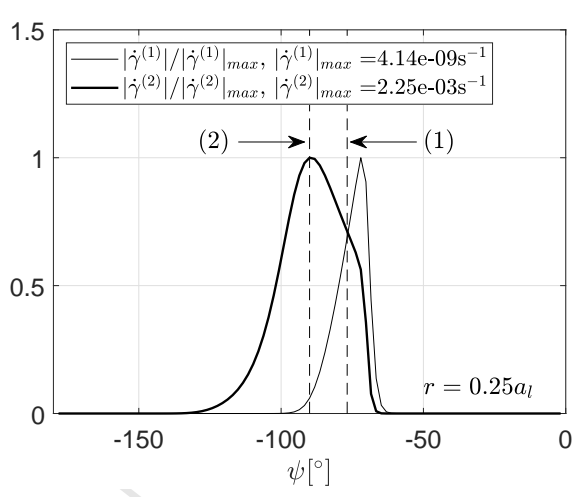

(b)

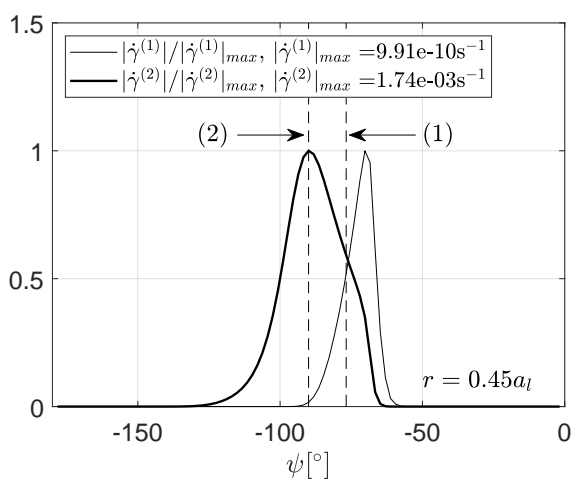

(d)

Figure 12: Angular slip rate distribution for monazite around the left contact point at a radius of; (a) $r=0.15 a_{l}$, (b) $r=0.25 a_{l}$, (c) $r=0.35 a_{l}$, and (d) $r=0.45 a_{l}$ away from the contact point. The dashed lines indicates the potential location of the slip rate discontinuity on slip system $(1)\left(-76.79^{\circ}\right)$ and slip system (2) $\left(-90^{\circ}\right)$. 


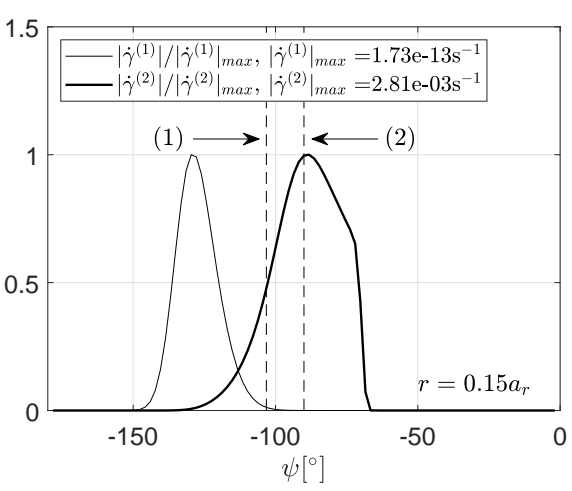

(a)

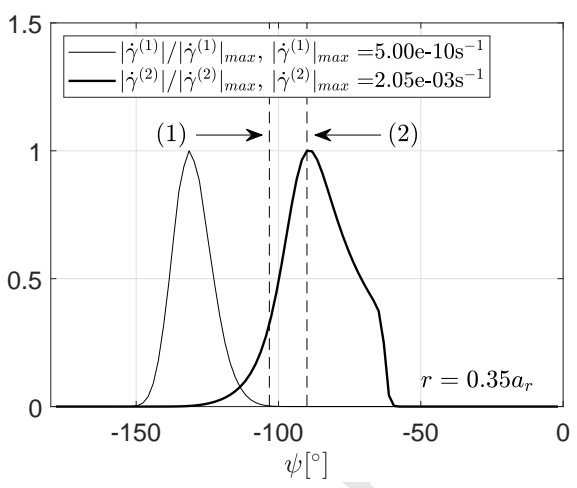

(c)

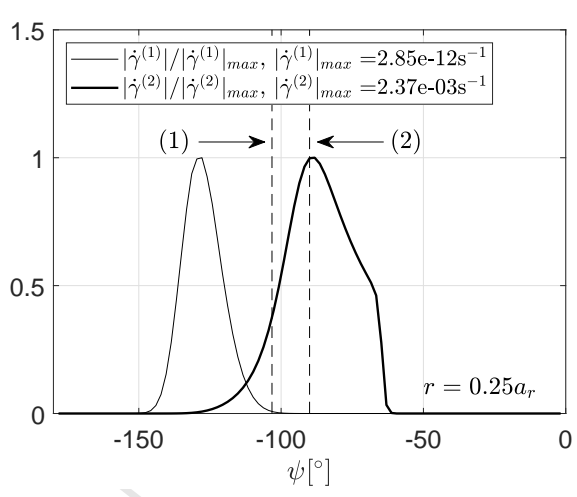

(b)

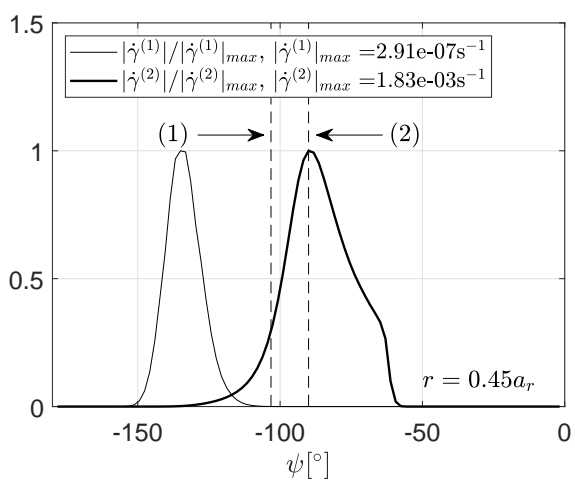

(d)

Figure 13: Angular slip rate distribution for monazite around the right contact point at a radius of; (a) $r=0.15 a_{r}$, (b) $r=0.25 a_{r}$, (c) $r=0.35 a_{r}$, and (d) $r=0.45 a_{r}$ away from the contact point. The dashed lines indicates the potential location of the slip rate discontinuity on slip system $(1)\left(-103.21^{\circ}\right)$ and slip system (2) $\left(-90^{\circ}\right)$. 


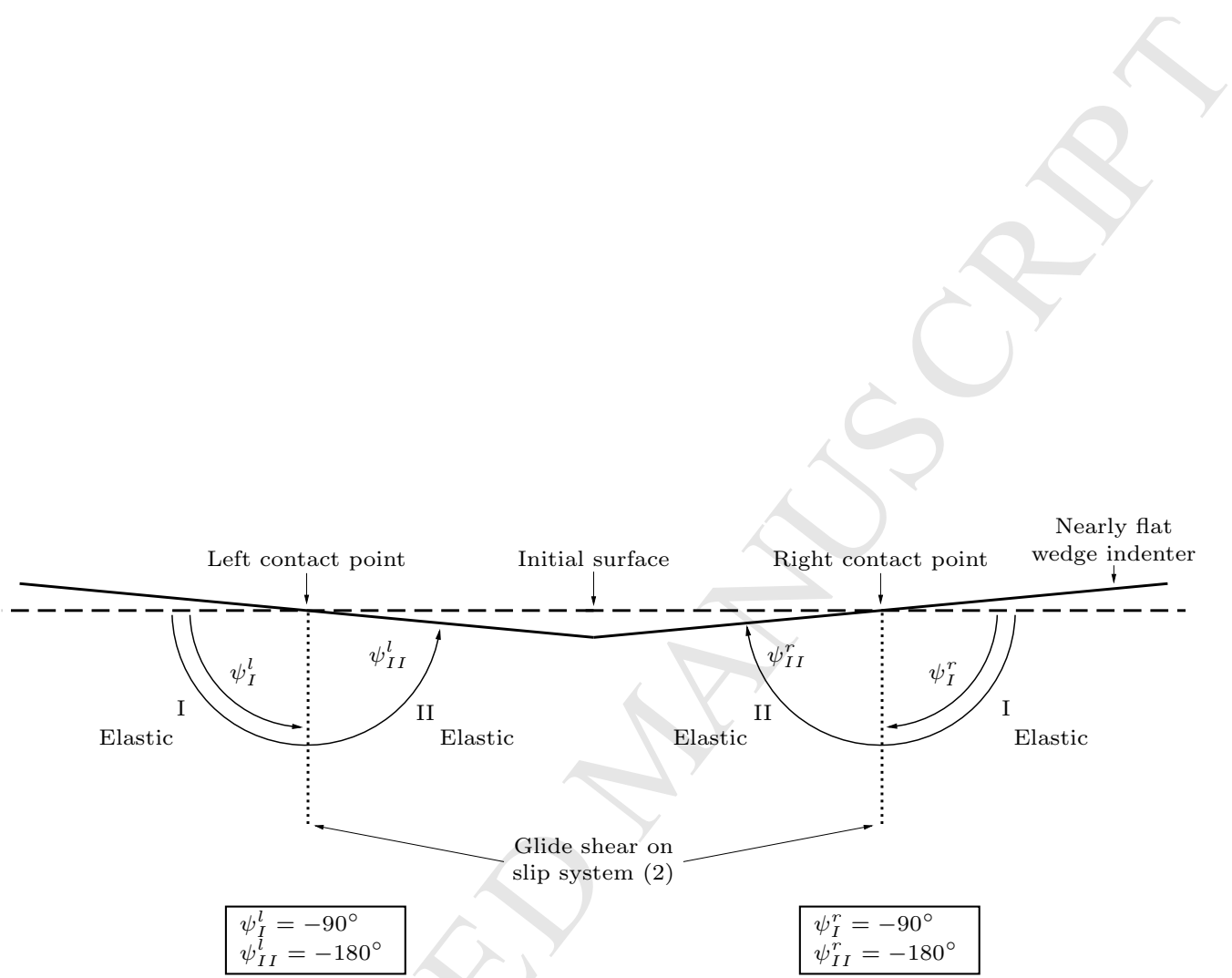

Figure 14: Numerically predicted sector structure in the vicinity of contact point singularities governed by an asymptotic solution. 
625

626

627

628

629

630

\section{List of Tables}

1 Model parameters. . . . . . . . . . . . . . . . . 50

2 Active slip systems in monazite at room temperature (identified by Hay, 2008). . . . . . . . . . . . . . . . . . . . . . . . . 51

3 In-plane slip systems of monazite. . . . . . . . . . . . . . 52

4 Vertices of the inner and outer envelope that make-up the yield surface of monazite (see Fig. 3) . . . . . . . . . . . . . . 53 


\begin{tabular}{cll}
\hline Parameter & Significance & Value \\
\hline$\tau_{0} / E$ & Yield strain & $\sim 4.5 \mathrm{e}^{-5}$ \\
$\nu$ & Poisson ratio & 0.3 \\
$m$ & Strain rate-sensitivity exponent & 0.02 \\
$\dot{\gamma}_{0}$ & Reference slip rate & $0.001 \mathrm{~s}^{-1}$ \\
$\dot{c}$ & Indentation rate & $0.5 \mathrm{~s}^{-1}$ \\
$\phi$ & Indenter angle & $0.038^{\circ}$ \\
\hline
\end{tabular}

Table 1: Model parameters. 


\begin{tabular}{cc}
\hline$\alpha$ & Slip System \\
\hline 1 & $(010)[001]$ \\
2 & $(010)[\overline{1} 00]$ \\
3 & $(\overline{1} 00)[010]$ \\
4 & $(\overline{1} 00)[001]$ \\
5 & $(\overline{1} \overline{1} 0)[001]$ \\
6 & $(\overline{1} \overline{1} 0)[\overline{1} 10]$ \\
7 & $(\overline{1} 10)[\overline{1} \overline{1} 0]$ \\
8 & $(\overline{1} 10)[001]$ \\
9 & $(011)[0 \overline{1} \overline{1}]$ \\
10 & $(011)[100]$ \\
11 & $(0 \overline{1} 1)[011]$ \\
12 & $(0 \overline{1} 1)[100]$ \\
\hline
\end{tabular}

Table 2: Active slip systems in monazite at room temperature (identified by Hay, 2008). 


\begin{tabular}{|c|c|c|c|}
\hline In-plane slip system (A) no. & (1) & $(2)$ & $(3)$ \\
\hline Mirrored pair of slip systems $\begin{array}{c}(\alpha) \\
(\beta)\end{array}$ & $\begin{array}{l}(011)[100] \\
(0 \overline{1} 1)[100]\end{array}$ & $\begin{array}{c}(100)[001] \\
-\end{array}$ & $\begin{array}{l}(\overline{1} \overline{1} 0)[001] \\
(\overline{1} 10)[001]\end{array}$ \\
\hline$s_{i}^{(\alpha)}=\left\{\begin{array}{l}s_{1} \\
s_{2} \\
s_{3}\end{array}\right\}^{(\alpha)}$ & $\left\{\begin{array}{c}0.9735 \\
0 \\
-0.2285\end{array}\right\}$ & $\left\{\begin{array}{l}0 \\
0 \\
1\end{array}\right\}$ & $\left\{\begin{array}{l}0 \\
0 \\
1\end{array}\right\}$ \\
\hline$m_{i}^{(\alpha)}=\left\{\begin{array}{l}m_{1} \\
m_{2} \\
m_{3}\end{array}\right\}^{(\alpha)}$ & $\left\{\begin{array}{l}0.1703 \\
0.6666 \\
0.7257\end{array}\right\}$ & $\left\{\begin{array}{c}-1 \\
0 \\
0\end{array}\right\}$ & $\left\{\begin{array}{c}-0.7281 \\
-0.6855 \\
0\end{array}\right\}$ \\
\hline$s_{i}^{(\beta)}=\left\{\begin{array}{l}s_{1} \\
s_{2} \\
s_{3}\end{array}\right\}$ & $\left\{\begin{array}{c}0.9735 \\
0 \\
-0.2285\end{array}\right\}$ & - & $\left\{\begin{array}{l}0 \\
0 \\
1\end{array}\right\}$ \\
\hline$m_{i}^{(\beta)}=\left\{\begin{array}{l}m_{1} \\
m_{2} \\
m_{3}\end{array}\right\}^{(\beta)}$ & $\left.\begin{array}{c}0.1703 \\
-0.6666 \\
0.7257\end{array}\right\}$ & - & $\left\{\begin{array}{c}-0.7281 \\
0.6855 \\
0\end{array}\right\}$ \\
\hline In-plane slip system & $(001)[100]$ & $(\overline{1} 00)[001]$ & $(\overline{100)}[001]$ \\
\hline$S_{I}^{(A)}=\left\{\begin{array}{l}S_{1} \\
S_{3}\end{array}\right\}^{(A)}$ & $\left.\begin{array}{c}0.9735 \\
-0.2285\end{array}\right\}$ & $\left\{\begin{array}{l}0 \\
1\end{array}\right\}$ & $\left\{\begin{array}{l}0 \\
1\end{array}\right\}$ \\
\hline$M_{I}^{(A)}=\left\{\begin{array}{l}M_{1} \\
M_{3}\end{array}\right\}$ & $\left\{\begin{array}{l}0.2285 \\
0.9735\end{array}\right\}$ & $\left\{\begin{array}{c}-1 \\
0\end{array}\right\}$ & $\left\{\begin{array}{c}-1 \\
0\end{array}\right\}$ \\
\hline Angle to $(100)$ in the $(010)$ plane: $\theta^{(A)}$ & $-13.21^{\circ}$ & $90^{\circ}$ & $90^{\circ}$ \\
\hline$\lambda^{(A)}=\frac{\tau_{0}^{(A)}}{\tau_{0}}=\frac{2}{\beta^{(A)}}$ & 1.3415 & 1 & 1.3735 \\
\hline$\beta^{(A)}=\frac{s_{I}^{(A \alpha)} m_{J}^{(A \alpha)}+s_{I}^{(A \beta)} m_{J}^{(A \beta)}}{S_{I}^{(A)} M_{J}^{(A)}}$ & 1.4909 & 1 & 1.4561 \\
\hline
\end{tabular}

Table 3: In-plane slị 2 systems of monazite. 


\begin{tabular}{ccc}
\hline Vertex & $\left(\sigma_{11}-\sigma_{33}\right) / 2 \tau_{0}$ & $\sigma_{13} / \tau_{0}$ \\
\hline $\mathrm{A}$ & 1.0022 & 1 \\
$\mathrm{~B}$ & -5.0277 & 1 \\
$\mathrm{C}$ & -1.0022 & -1 \\
$\mathrm{D}$ & 5.0277 & -1 \\
$\mathrm{E}$ & 0.2505 & 1.3735 \\
$\mathrm{~F}$ & -5.7794 & 1.3735 \\
$\mathrm{G}$ & -0.2505 & -1.3735 \\
$\mathrm{H}$ & 5.7794 & -1.3735 \\
\hline
\end{tabular}

Table 4: Vertices of the inner and outer envelope that make-up the yield surface of monazite (see Fig. 3). 
- Modelling of wedge indentation in a monazite single crystal (monoclinic structure).

- Mapping of material behavior in the vicinity of the contact point singularities.

- Presentation of the field distributions in the vicinity of the contact point singularities.

- Identification of individual velocities of the contact point singularities. 